\title{
A study of hydride precipitation in zirconium
}

\author{
I.A. Khan ${ }^{1}$, A.C.F. Cocks $^{2}$, J. Chattopadhyay ${ }^{1}$ \\ ${ }^{1}$ Reactor Safety Division, Bhabha Atomic Research Centre, \\ Trombay, Mumbai-400085, India \\ ${ }^{1}$ Department of Engineering Science, University of Oxford, \\ Parks Road, OX1 3PJ, United Kingdom
}

January 12, 2021

\begin{abstract}
Zirconium-based alloys used in nuclear reactors are susceptible to hydrogen pickup. When the hydrogen concentration in the solid solution exceeds the threshold limit (terminal solubility), a solid-state phase transformation occurs in these alloys. The zirconium hydride phase so precipitated is brittle and may influence the integrity of in-core structural components by various mechanisms, such as hydride blistering, delayed hydride cracking (DHC) etc. Precipitation which usually occurs by nucleation, growth and coarsening is typically influenced by the coherent stresses as well as the external stress field. In this work, Eshelby-type analyses are carried out to understand the mechanics and energetics associated with precipitation of zirconium hydrides. In particular, the role of elastic mismatch between the matrix and inclusion and the external stress in controlling the morphology and orientation of a hydride precipitate is analyzed. Elastic calculations lead to several interesting findings and reveal that under the influence of an external stress, a hydride precipitate has a tendency to reorientate along a certain crystallographic plane of the zirconium matrix. Incorporation of hydride plasticity, in our proposed scheme, leads to hydride reorientation at realistic magnitudes of applied stress. The strong anisotropy in interfacial energy, however, causes a hydride precipitate to remain parallel to the basal plane. Potential applications of our proposed numerical scheme to other relevant fields are also discussed.
\end{abstract}

Keywords: Phase transformation, zirconium alloys, hydride reorientation, Eshelby analysis.

\section{Introduction}

Zirconium-based alloys possess a number of desirable properties that make them of immediate interest to the nuclear industry. Among these, the low absorption cross-section for neutrons, good corrosion resistance in steam/water environments and adequate strength make them suitable for in-core structural applications. When hydrogen concentration in zirconium alloys exceeds the terminal solid solubility, it precipitates out as a zirconium hydride phase. Based 
on hydrogen content, cooling rate and temperature of precipitation, $\delta, \gamma$ or $\varepsilon$-hydride can form (Northwood and Kosasih, 1983). For the cooling rates typically encountered by the pressure tubes used in CANDU-type reactors, the $\delta$-hydride phase with a platelet morphology is formed. Recent experimental investigations on Zircaloy-4 also showed that for the range of temperature and hydrogen concentrations relevant to light water reactors (LWRs), only $\delta$-hydride is formed (see Bair et al., 2015). The $\delta$-hydride phase so precipitated is brittle and has a substantial influence on the mechanical properties of zirconium alloys. In particular, the reduction in ductility can lead to failure of structural components by various mechanisms, such as pellet clad mechanical interactions and hydride blistering. It is, however, the mechanism of delayed hydride cracking (DHC) that is of much concern in zirconium alloys (Puls, 2012). DHC is a time-dependent phenomenon in which the repeated formation of zirconium hydride at the tip of a crack leads to embrittlement of the matrix. The degree of embrittlement is strongly influenced by the morphology and orientation of platelets relative to the external stress (see Northwood and Kosasih, 1983; Bair et al., 2015). It has been reported that hydride orientation depends on the crystallographic texture of the matrix, prior cold-working and the applied stress (Northwood and Kosasih, 1983). In order to retain sufficient ductility, the fabrication route for structural components like fuel clad and pressure tubes is tailored such that predominantly circumferential hydrides are formed during the service conditions. Radial hydrides can, however, form by a reorientation process when a specimen is cooled down under tensile stress from a temperature at which hydrides are dissolved. Many investigators have attempted to analyze this problem. Ells (1970) has examined the reorientation of a hydride nucleus without incorporating the strain energy resulting from hydride precipitation. The models of Puls (2009) and Bai et al. (1994) overcome this limitation but none of them accounts for the effect of inhomogeneity (mismatch in elastic moduli of a precipitate and matrix) on the total potential energy. Chu et al. (2008) incorporated an empirical constant, determined from fitting experimental data, in their model.

In addition to the analytical/empirical models described above, extensive computational modeling studies have been carried out to understand hydrogen diffusion, trapping and hydride precipitation in zirconium at different length scales. Atomic scale simulations employing Density Function theory (DFT) and Molecular Dynamics (MD) have provided useful insights on the hydrogen transport, hydride nucleation and the properties of nanoscale hydride phases, (see Motta et al., 2019). Since a phase field model can incorporate several thermodynamic driving forces like the bulk and interfacial energies, elastic strain energy, plastic deformations etc., it has been widely used to study the microstructure evolution of zirconium hydride phases at the mesoscale. A detailed account of phase field studies on hydride precipitation in zirconium alloys can be found in the works of Bair et al. (2015) and Motta et al. (2019). Quite recently, phase field studies analyzing the morphology and reorientation of hydride precipitates have also been reported, (see Bair and Zaeem, 2017; Han et al., 2019).

Nucleation of precipitates in solids is mostly of heterogeneous type with defects such as vacancies, dislocations, grain boundaries etc. as the preferred sites (Christian, 2002). For heterogeneous nucleation, the change in Gibbs free energy can be expressed as,

$$
\Delta G_{H e t}=-V\left(\Delta G_{V}-\Delta G_{S}\right)+A \gamma-\Delta G_{d}
$$

Here, $\Delta G_{V}$ is the free energy change per unit volume caused by the precipitation of a new phase often referred as the chemical energy, $\Delta G_{S}$ is the elastic strain energy per unit volume 
due to lattice misfit, $\gamma$ is the interfacial energy, $\Delta G_{d}$ is the energy released from a defect due to creation of a nucleus, $V$ is the volume of precipitate and $A$ is the interfacial area between the matrix and the precipitate. The combination of size, shape and spatial distribution of a nucleus that leads to a stationary value of $\Delta G_{H e t}$ dictates the morphology and orientation of the new phase (Christian, 2002). For a precipitate that is sufficiently large in comparison to the critical-sized nucleus, the contribution from interfacial energy is much smaller in comparison to that of the strain energy. Most of our calculations, therefore, do not account for the effect of interfacial energy. In some cases, the interfacial energy, however, plays a significant role in the energetics of precipitation, (see Gill and Cocks, 2006; Massih and Jernkvist, 2009; Han et al., 2019). For an evolving microstructure, typically both the chemical energy and the elastic strain energy control the morphology and orientation of the precipitated phase. The question that is posed here is for a given volume of a precipitate, what is its shape and orientation that is preferred energetically? If the chemical energy of the new phase, per unit volume, is treated as a constant then for a given volume of a precipitate it is only the elastic strain energy that is a function of precipitate's shape and its orientation. For such a case, the effect of shape and orientation of a precipitate on the Gibbs free energy, thus, can be solely analyzed in terms of mechanical energy, (see Sharma et al., 2018).

Typically, the elastic strain energy arising from lattice distortions such as dislocations, precipitates and inhomogeneities is calculated using the Eshelby theory (Eshelby, 1957). Mori et al. (1978) utilized this approach to study the effect of shape and orientation of an inhomogeneous inclusion on the strain energy, for a face-centred-cubic copper matrix. Transformation (eigen) strains were assumed to be purely dilatational. Similar analyses were carried out by Sato et al. (1979) to compute the disturbance in the stress field around a spherical inhomogeneity embedded in a Fe-Ni single crystal. The effect of precipitate shape and orientation on the elastic strain energy of an inhomogeneous inclusion with deviatoric misfit strains was studied by Onaka et al. (1995). Similar detailed studies analyzing the energetics of hydride precipitation in zirconium alloys are, however, lacking. Based on elastic interaction energy, Perovik et al. (1981) explain the autocatalytic nucleation events where the stress field of the larger hydride assists in nucleation of the daughter hydride. Leitch and Puls (1992) performed finite element studies to compute elastic-plastic accommodation energy for various oblate shaped precipitates. The effect of external stress and inhomogeneity was not accounted for. Singh et al. (2008) carried out a similar study on a thin circular disc to predict the habit plane for $\delta$-hydride precipitating in an $\alpha$-zirconium matrix.

In the present work, a numerical scheme based on Eshelby's solution is developed to analyze an inhomogeneous inclusion in an anisotropic elastic medium. In particular, the role of elastic mismatch between the matrix and inclusion and external stress in controlling the morphology and orientation of a single hydride precipitate is examined. The effects of applied stress direction with respect to matrix crystallographic planes and stress biaxiality on the orientation of a hydride precipitate are studied. For the case of an isotropic matrix and dilatational eigenstrains, closed-form expressions describing the effect of elastic mismatch and external stress on the orientation of a disc and needle shaped precipitate are proposed. These elastic calculations provide insights into the factors controlling the mechanics of hydride reorientation. Numerical solutions indicate that a hydride precipitate under an external stress has a tendency to reorientate along a certain crystallographic plane of the zirconium matrix. The assumption of linear elasticity, however, leads to a large overestimation of the external stress required for hydride reorientation. To overcome this limitation, a numerical scheme that incorporates elastic-plastic 
deformation of a hydride precipitate is developed. The matrix response is still linear elastic. Incorporation of hydride plasticity in our proposed scheme leads to reorientation of a hydride precipitate at realistic magnitudes of applied stress. Experimental evidence, however, suggests that under an external stress an individual hydride precipitate remains parallel to the basal plane (see Motta et al., 2019; Han et al., 2019). It is the stacking arrangement of microscopic hydrides that gives an appearance of reoriented macrohydrides typically observed under an optical microscope. Incorporation of interfacial energy in our numerical calculations reveals that for a small hydride the anisotropy in surface energy controls the orientation of a precipitate, thus, causing it to remain parallel to the basal plane.

\section{Numerical scheme for an inhomogeneous inclusion in anisotropic elastic medium}

Eshelby (1957) developed an elegant scheme to determine the elastic field of an ellipsoidal inclusion in an isotropic infinite medium. It is assumed that a region within an isotropic elastic solid undergoes a spontaneous change of form which, in the absence of a surrounding material, would lead to some prescribed homogeneous deformation. The presence of surrounding material will, however, constrain this free expansion or contraction and, thus, generate stresses both inside and outside the region of transformation. In particular, if the region is an ellipsoid, the total (constrained) strains $\gamma_{i j}$ inside it are uniform and can be expressed as (Eshelby, 1957),

$$
\gamma_{i j}=S_{i j m n} \varepsilon_{m n}^{p}
$$

Here, $\varepsilon_{m n}^{p}$ are the prescribed transformation strains that are assumed to be uniform inside the ellipsoidal domain, and $S_{i j m n}$ are the components of the fourth-order Eshelby tensor. Closedform solutions for $S_{i j m n}$ are available only for isotropic, and transversely isotropic media. Since an explicit expression for Green's function, on which Eshelby analysis is based, is not available for a general anisotropic medium, Mura and co-workers (1987) performed integrations in Fourier and physical space to compute the components of the Eshelby tensor. The resulting expression at an arbitrary point within the inclusion in an anisotropic elastic medium is as follows (Mura, 1987),

$$
S_{i j m n}=\frac{1}{8 \pi} C_{p q m n} \int_{-1}^{1} d \bar{\zeta}_{3} \int_{0}^{2 \pi}\left\{G_{i p j q}(\bar{\xi})+G_{j p i q}(\bar{\xi})\right\} d \theta
$$

Here, $C_{p q m n}$ are the elastic moduli of the anisotropic matrix, $\bar{\xi}$ is the unit vector and the integration is carried out on a unit sphere. Tensor components $G_{i j k l}(\bar{\xi})$ are defined as (Mura, 1987),

$$
G_{i j k l}(\bar{\xi})=\bar{\xi}_{k} \bar{\xi}_{l} N_{i j}(\bar{\xi}) / D(\bar{\xi})
$$

$N_{i j}(\bar{\xi})$ and $D(\bar{\xi})$ are, respectively, the cofactors and determinant of the matrix $K$, whose components are

$$
K_{i k}(\bar{\xi})=C_{i j k l} \bar{\xi}_{j} \bar{\xi}_{l}
$$

Explicit expressions for $N_{i j}(\bar{\xi})$ and $D(\bar{\xi})$ are 


$$
N_{i j}(\bar{\xi})=\frac{1}{2} \epsilon_{i k l} \epsilon_{j m n} K_{k m} K_{l n}, \quad D(\bar{\xi})=\epsilon_{m n l} K_{m 1}(\bar{\xi}) K_{n 2}(\bar{\xi}) K_{l 3}(\bar{\xi})
$$

Here $\epsilon_{i j k}$ is the standard permutation tensor.

The components of $(\bar{\zeta})$ are defined as,

$$
\bar{\zeta}_{1}=\left(1-\bar{\zeta}_{3}^{2}\right)^{\frac{1}{2}} \cos \theta, \quad \bar{\zeta}_{2}=\left(1-\bar{\zeta}_{3}^{2}\right)^{\frac{1}{2}} \sin \theta, \quad \bar{\zeta}_{3}=\bar{\zeta}_{3}
$$

The relations between $\bar{\xi}_{i}$ and $\bar{\zeta}_{i}$ can be expressed as,

$$
\bar{\xi}_{1}=\frac{\bar{\zeta}_{1}}{a_{1}}, \quad \bar{\xi}_{2}=\frac{\bar{\zeta}_{2}}{a_{2}}, \quad \bar{\xi}_{3}=\frac{\bar{\zeta}_{3}}{a_{3}}
$$

$a_{i}$ are the lengths of semi-axes of an ellipsoidal inclusion. The scheme described above is in terms of a double quadrature that needs to be evaluated numerically. Numerical implementation is, however, not straightforward. Moreover, the mismatch in elastic moduli of a precipitate $C_{i j k l}^{*}$ and matrix makes the problem quite involved. For such cases, Eshelby's equivalent inclusion method (1957) is often employed to compute the equivalent eigenstrains $\varepsilon_{m n}^{* *}$. The calculation is based on the equivalence of stress and strain fields of the inhomogeneous inclusion with that of a corresponding homogeneous one. This essentially involves the solution of the following system of linear equations (Mura, 1987),

$$
\sigma_{i j}^{o}+\sigma_{i j}=C_{i j k l}^{*}\left(\varepsilon_{k l}^{o}+S_{k l m n} \varepsilon_{m n}^{* *}-\varepsilon_{k l}^{p}\right)=C_{i j k l}\left(\varepsilon_{k l}^{o}+S_{k l m n} \varepsilon_{m n}^{* *}-\varepsilon_{k l}^{* *}\right)
$$

Here $\sigma_{i j}^{o}$ is the remotely applied stress in a material that is free from any inclusion. $\sigma_{i j}$ is the sum of stress caused by the inhomogeneity and the internal stress arising from the eigenstrains. The fictious eigenstrains $\varepsilon_{m n}^{*}$ introduced in the equivalent inclusion method can be obtained as,

$$
\varepsilon_{i j}^{*}=\varepsilon_{i j}^{* *}-\varepsilon_{i j}^{p}
$$

The total elastic strain energy of a precipitate and matrix $W^{*}$ can be obtained from the following expression (Mura, 1987),

$$
W^{*}=-\frac{1}{2} \int_{\Omega} \sigma_{i j} \varepsilon_{i j}^{p} d V+\frac{1}{2} \int_{\Omega} \sigma_{i j}^{o} \varepsilon_{i j}^{*} d V
$$

Here, $\Omega$ is the volume of a precipitate. For the reasons discussed earlier, the interfacial energy and the chemical energy do not enter in our calculations and, therefore, the Gibbs free energy of the body can be expressed as (Mura, 1987),

$$
\begin{aligned}
& G=G^{o}+G^{*}=\left(\frac{1}{2} \int_{D} \sigma_{i j}^{o} \varepsilon_{i j}^{o} d V-\int_{S} F_{i} u_{i}^{o} d S\right)+ \\
& \left(-\frac{1}{2} \int_{\Omega} \sigma_{i j} \varepsilon_{i j}^{p} d V-\int_{\Omega} \sigma_{i j}^{o} \varepsilon_{i j}^{p} d V-\frac{1}{2} \int_{\Omega} \sigma_{i j}^{o} \varepsilon_{i j}^{*} d V\right)
\end{aligned}
$$

Here, $G^{o}$ is the Gibbs free energy (total potential energy) of a homogeneous body without an inclusion. $G^{*}$ represents the sum of three terms which, following the notation of Pineau (1976), will be referred as the inclusion energy, interaction energy and the energy of inhomogeneity, respectively. $D$ is the volume of a body containing an inhomogeneous inclusion. $u_{i}^{o}$ is the 


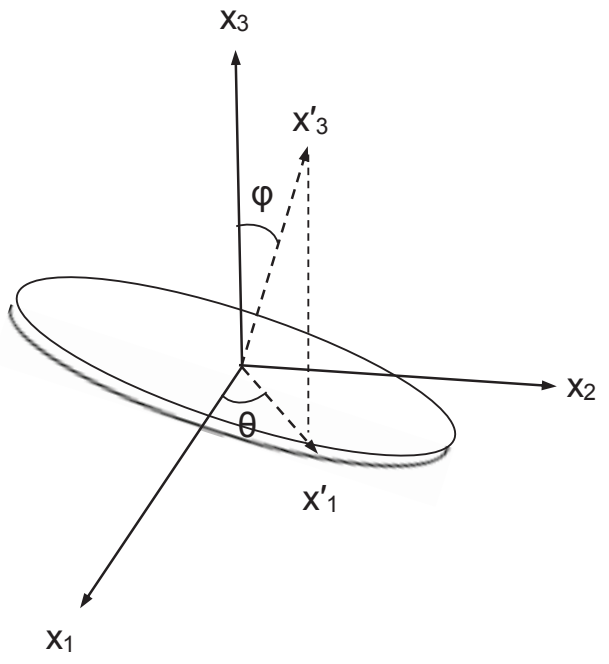

(a)

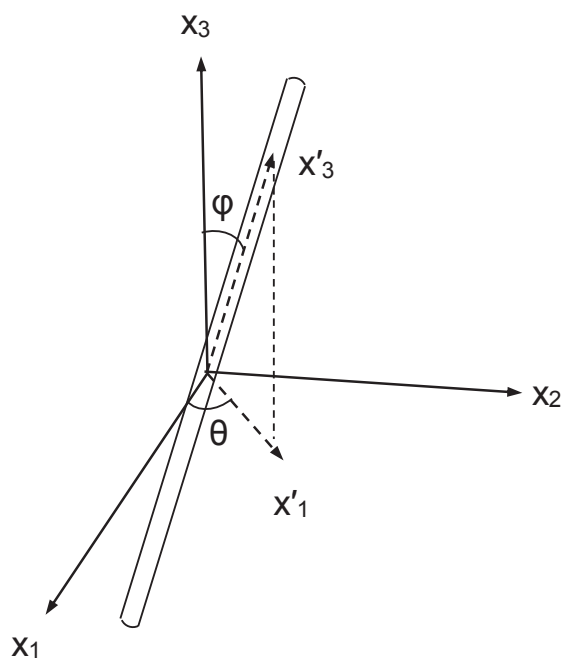

(b)

Figure 1: Orientation of (a) disc and (b) needle shaped precipitate with respect to matrix crystallographic directions $x_{i}$.

displacement due to external stress and $F_{i}=\sigma_{i j}^{o} n_{j}$ is the traction on the exterior boundary of $D$.

In order to account for the orientation of a precipitate with respect to matrix crystallographic directions, see fig.1, calculations are carried out in a local Cartesian coordinate system. The origin of the local system coincides with the centre of the ellipsoid and the three principal axes of the ellipsoid are aligned with the local Cartesian axes $\dot{x}_{i}$. The components of the rotation matrix $R_{i j}$, describing the cosine of angles between the matrix crystallographic directions $x_{i}$ and the local axes of the precipitate $x_{j}^{\prime}$, are as follows,

$$
R_{i j}=\left[\begin{array}{ccc}
\cos \theta \cos \phi & \sin \theta \cos \phi & -\sin \phi \\
-\sin \theta & \cos \theta & 0 \\
\cos \theta \sin \phi & \sin \theta \sin \phi & \cos \phi
\end{array}\right]
$$

Transformation of the respective tensors of elastic moduli of the matrix and the precipitate, $C_{i j k l}$ and $C_{i j k l}^{*}$, and the prescribed eigenstrain tensor $\varepsilon_{i j}^{p}$ is carried out as per the standard equations,

$$
\begin{gathered}
C_{i j k l}^{\prime}=R_{i p} R_{j q} R_{k r} R_{l s} C_{p q r s} \\
C_{i j k l}^{*}=R_{i p} R_{j q} R_{k r} R_{l s} C_{p q r s}^{*} \\
\varepsilon_{i j}^{p}=R_{i p} R_{j q} \varepsilon_{p q}^{p}
\end{gathered}
$$

The numerical scheme described above is implemented in a FORTRAN computer program. The double integral appearing in eq.(3) is evaluated using the Gauss quadrature rule. The minimum number of Gauss points required for an accurate evaluation of the Eshelby's tensor 
components $S_{i j m n}$ depends on the degree of matrix anisotropy and the aspect ratio of the ellipsoidal inclusion. All the numerical results presented in this study are obtained using $64 \mathrm{x}$ 64 Gauss points for integration over $\bar{\zeta}_{3}$ and $\theta$ space to ensure numerical accuracy. The system of linear equations, eq.(9), is solved simultaneously using a LU decomposition technique followed by backward substitution. Numerical implementation is validated by comparing our predictions with previously published results (Mori et al., 1978; Sato et al., 1979; Mura, 1987).

\section{Elastic inhomogeneous inclusion in an isotropic matrix}

Before proceeding to the problem of hydride precipitation in zirconium, we begin with an analysis of an elastic inhomogeneous inclusion in an isotropic matrix. This problem is relatively simple as, for the case of dilatational eigenstrains, the direction of external stress relative to the inclusion is the only factor that can influence its orientation. The terminology used is consistent with the one adopted by Mura (1987). When the elastic moduli of an ellipsoidal sub-domain of a material differ from the matrix, it is referred as an "inhomogeneity". An "inclusion" has the same elastic moduli as the matrix but contains eigenstrains. When an inhomogeneity contains an eigenstrain, it is called an "inhomogeneous inclusion". Typically, most of the precipitates in alloys and martensite, formed during phase transformation, are inhomogeneous inclusions.

The problem of an inhomogeneous inclusion in an isotropic elastic matrix, under an external stress, has been analyzed in the past (Pineau, 1976). Eigenstrains were assumed to be purely dilatational. Three different cases namely; a sphere, a disc perpendicular to an applied stress and a needle aligned with the stress axis were considered. The influence of elastic mismatch between the matrix and precipitate, the particle shape and magnitude of uniaxial applied stress on the elastic strain energy was analyzed. Unfortunately, as will be demonstrated in this section, such simple orientations are not always the configurations of minimum potential energy.

We present here, closed-form expressions to describe the effect of elastic mismatch between the matrix and precipitate and the dilatational eigenstrains on the total potential energy of disc and needle shaped precipitates and their preferred orientation under a uniaxial applied stress, see Appendix-A. Both the precipitate and matrix are assumed to be elastically isotropic. The Young's modulus of the precipitate can be different from that of the matrix, the ratio is described by a scalar factor $f$. Poisson's ratio $\nu$ of the precipitate and the matrix are, however, assumed to be the same. The numerical results presented in this section are obtained using $\nu=0.3$. Elastic strain/potential energy is calculated per unit volume of precipitate and the volume fraction is assumed to be small so that the elastic interactions between the neighboring particles can be ignored. While determining the total potential energy, the system comprising of a matrix, an ellipsoidal precipitate and an external stress is considered. Following Pineau (1976), the initial state is taken as a loaded system still in solid solution while the final state comprises the loaded system containing precipitates of different shapes and orientations. In our numerical results, the total potential energy is always with respect to the initial loaded state.

\subsection{Orientation of an elastic inhomogeneity under an external stress}

To gain insights into the energetics leading to some preferred orientation of a precipitate, it will be advantageous to first analyze the pure inhomogeneity problem. Since the eigenstrains are zero, the effect of precipitate orientation on the total potential energy is solely a consequence 
of interaction between the applied stress and the perturbation resulting from the elastic field of an inhomogeneity.

We consider here two simple shapes of precipitates namely; a disc and a needle subjected to a uniaxial applied stress. For all the numerical results presented in this article, a disc is defined as an oblate spheroid with an aspect ratio $c / a \approx 10^{-4}$ whereas a needle is defined as a prolate spheroid with $c / a \approx 10^{4}$. Here, $c$ and $a$ are the semi-minor and semi-major axes of an ellipsoid. For such idealized configurations, closed-form solutions of a precipitate's orientation leading to minimum potential energy are developed in Appendix-A. These preferred orientations are independent of the nature (tensile/compressive) and magnitude of applied stress. For a disc shaped inhomogeneity in an isotropic elastic matrix, the orientation corresponding to minimum potential energy is given by the following equation (see Appendix A.2),

$$
\tan \phi=\sqrt{\frac{\nu}{1-\nu}}
$$

Here, $\nu$ is Poisson's ratio of the matrix. Thus, the preferred orientation of a thin disc is independent of the elastic mismatch between the matrix and precipitate. For further clarity, we plot here the variation of total potential energy $G^{*}$ of a disc shaped elastic precipitate arising from normal and shear components of applied stress, as a function of precipitate orientation, see fig. 2 .

Two different values of elastic mismatch factor $f=0.5$ and 1.5 are considered. For an undermatched precipitate $(f<1)$, the negative values of $G^{*}$ simply means that the total potential energy is less than that of the reference configuration. The applied shear stress, inside a precipitate, becomes maximum for $\phi=45^{\circ}$. The total potential energy from applied shear stress, therefore, attains an extremum at this orientation. The variation of total potential energy resulting from the normal components of applied stress is, however, asymmetric. For $\nu=0.3$, the orientation corresponding to minimum potential energy, eq.(17), is $33.2^{\circ}$.

For a needle shaped precipitate, the orientation corresponding to minimum potential energy can be obtained from the following equation (Appendix-A.3),

$$
\tan \phi=\sqrt{\frac{-(1+f-2 \nu)}{\bar{D}}}
$$

Here,

$$
\bar{D}=\frac{4 f^{2} \nu^{2}-3 f^{2} \nu-3 f \nu+f+4 \nu^{2}-4 \nu+1}{3 f-4 f \nu+1}
$$




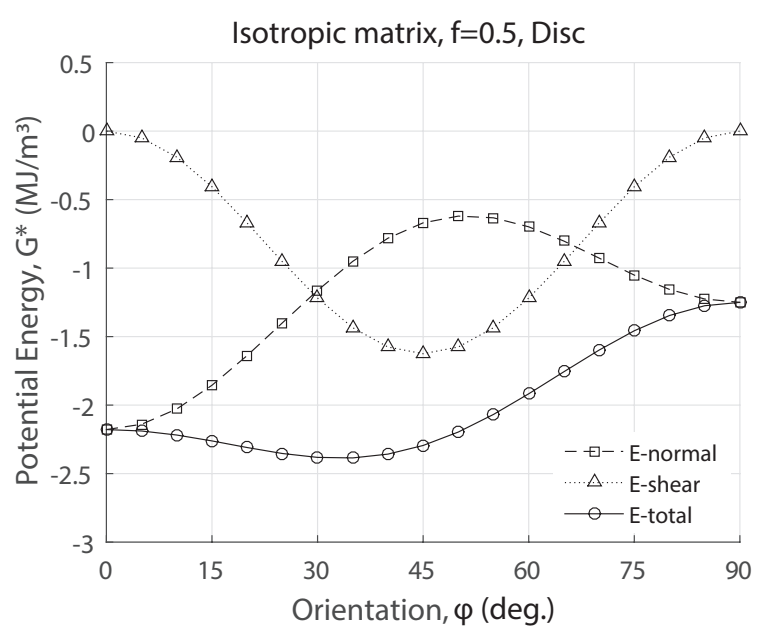

(a)

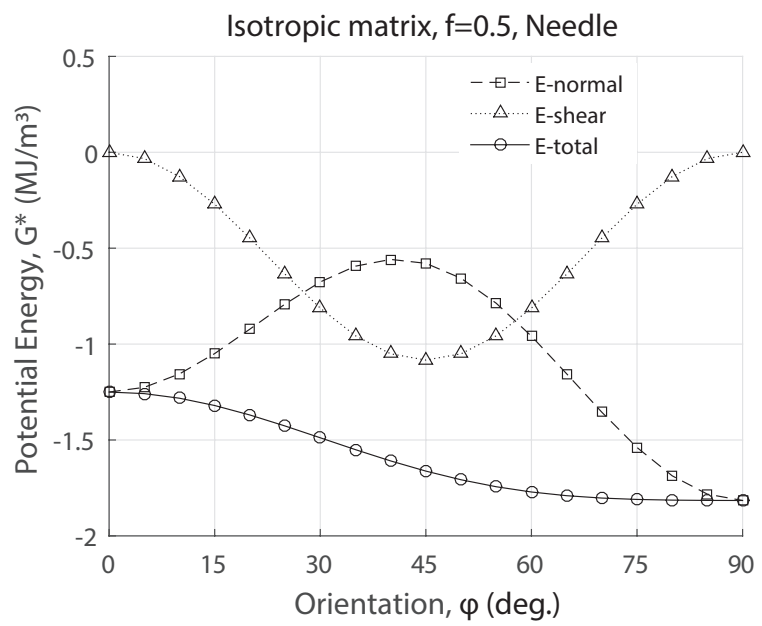

(c)

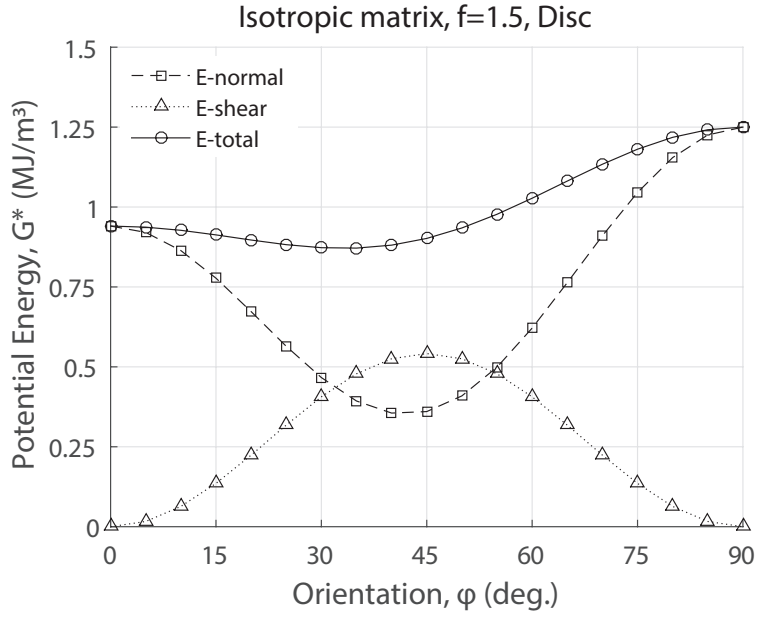

(b)

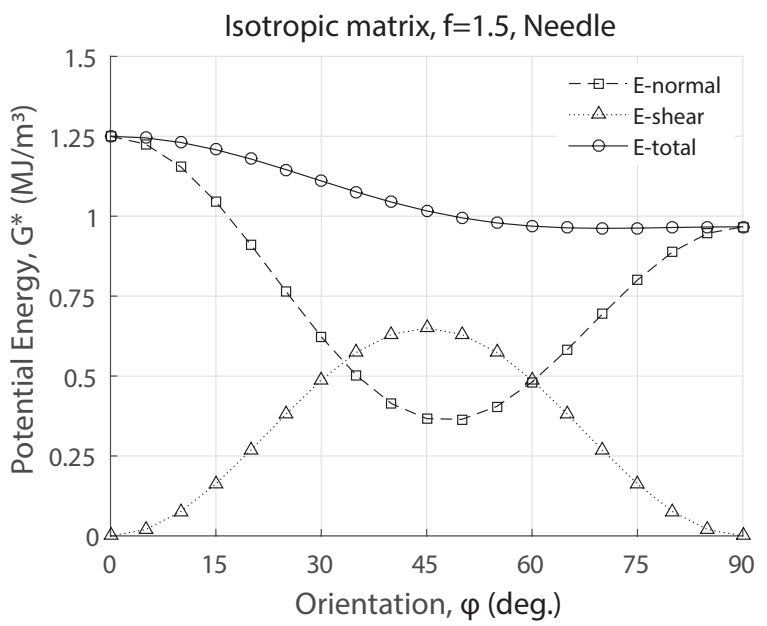

(d)

Figure 2: Total potential energy $G^{*}$ of a disc shaped elastic inhomogenety (eigenstrains are zero) as a function of orientation angle $\phi$ for an elastic mismatch factor, (a) $f=0.5$ and (b) $f=1.5$, respectively. Corresponding results for a needle shaped precipitate are shown in (c) and (d), respectively. Numerical results are presented for an applied stress of 1000 MPa. Notations $\mathrm{E}_{\text {normal }}$ and $\mathrm{E}_{\text {shear }}$ represent the potential energy resulting from the normal and shear components of the applied stress, respectively. The sum of these two energies $\left(\mathrm{E}_{\text {total }}\right)$ is numerically equal to $G^{*}$

Unlike the case of a disc, the orientation minimizing the total potential energy of a needle shaped precipitate is a complicated function of the elastic mismatch factor $f$ and Poisson's ratio $\nu$. The variation of total potential energy $G^{*}$ arising from normal and shear components of applied stress, for a needle shaped precipitate is shown in fig.2(c) and (d). For the case of a needle the orientation angle $\phi$ minimizing the potential energy, obtained from eq.(18), increases as the mismatch factor $f$ decreases and attains a value of $90^{\circ}$ at $f=0.645$. When $f \leq 0.645$, no real analytical solution exists for the orientation that minimizes the potential energy. Numerical solutions suggest that the orientation attained at $f=0.645$ is maintained as $f$ further decreases, 
that is, for $f \leq 0.645$ a needle perpendicular to the applied stress is the configuration of minimum potential energy. It may also be noted that as $f \rightarrow \infty, \phi \rightarrow 61.2^{\circ}$. For $f=1.5$, the minimum potential energy occurs at $\phi=70.2^{\circ}$.

\subsection{Morphology and orientation of an elastic inhomogeneous inclu- sion under an external stress}

We consider here, a more general case where the inhomogeneity has its own eigenstrain. For simplicity, the eigenstrain is assumed to be purely dilatational. We first analyze the effect of elastic mismatch factor $f$ on the normalized strain energy $W^{*} / E \delta^{2}$ for three different shapes of precipitate namely disc, sphere and needle, see fig.3.

$E$ is the elastic modulus of the matrix and $\delta$ is the isotropic eigenstrain. The external stress is assumed to be zero. Our numerical results seem to be in very good agreement with those of Pineau (1976). For a homogeneous inclusion $(f=1)$, the elastic strain energy becomes independent of its shape (Eshelby, 1957). It is worth noting that the in-plane components of equivalent eigenstrains and, hence, the elastic stresses inside a disc shaped precipitate scale linearly with the mismatch factor $f$. This is precisely the reason for linear variation of elastic strain energy with $f$ for a thin disc. As reported by Pineau (1976), for an undermatched case $(f<1)$, a thin disc leads to minimum strain energy whereas for an overmatched case, a sphere is the most preferred shape.

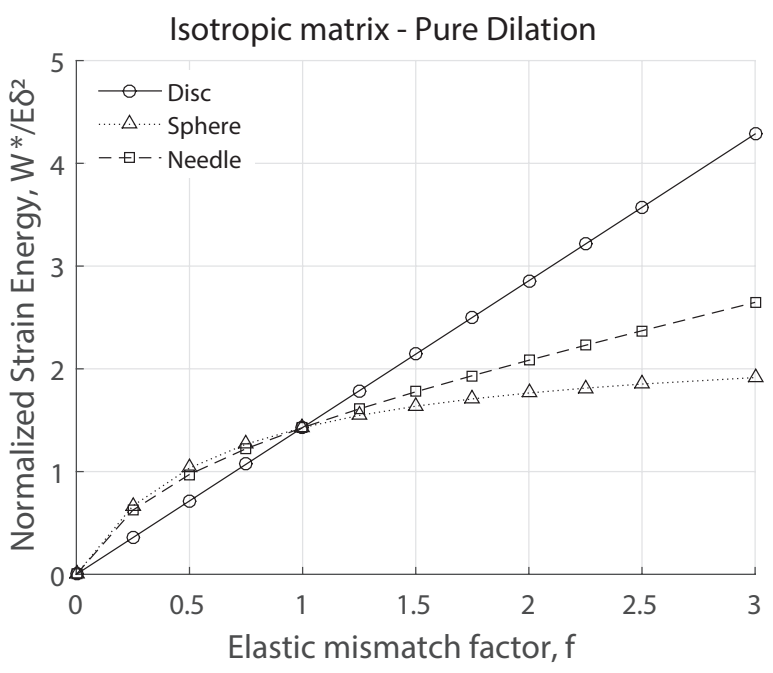

Figure 3: Elastic strain energy $W^{*}$ as a function of mismatch factor $f$ for three different shapes of precipitate. Eigenstrain $\delta$ is assumed to be purely dilatational.

We now proceed to the case of an inhomogeneous inclusion subjected to a uniaxial stress (say, $\sigma_{33}^{o}$ ). The total potential energy is calculated as a function of precipitate shape, the applied stress and the elastic mismatch factor $f$. For comparison, the case of a disc perpendicular to the applied stress and a needle aligned with the stress axis, considered by Pineau (1976), are also analyzed, see fig.4. Among the various shapes, the one that minimizes the total potential energy corresponds to the preferred configuration (surface energy consideration not being taken into account). 


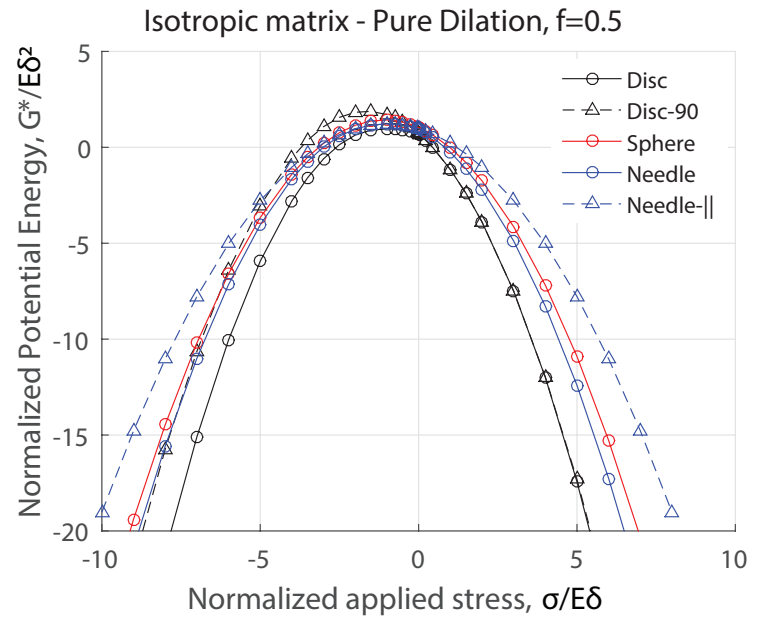

(a)

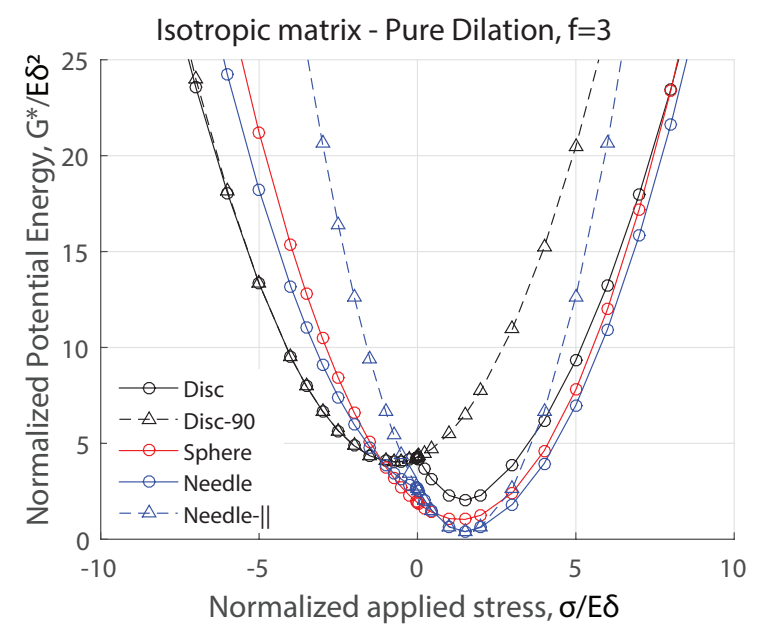

(c)

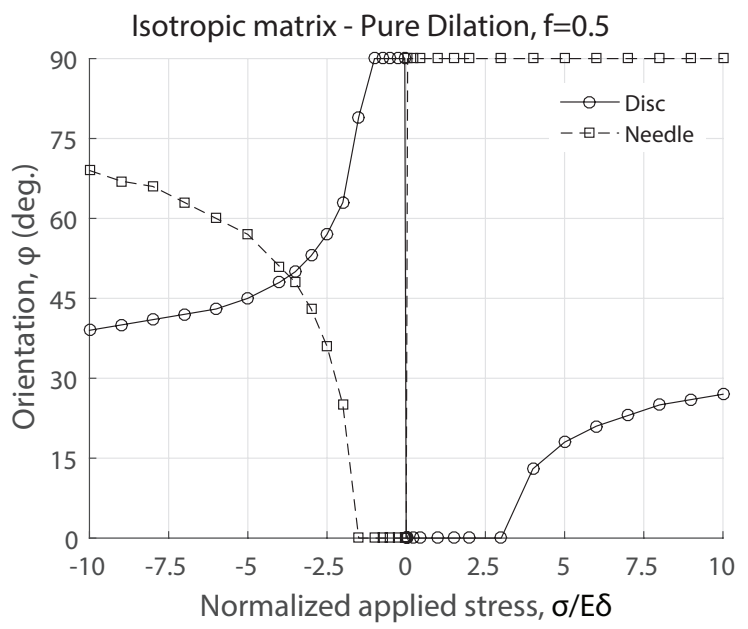

(b)

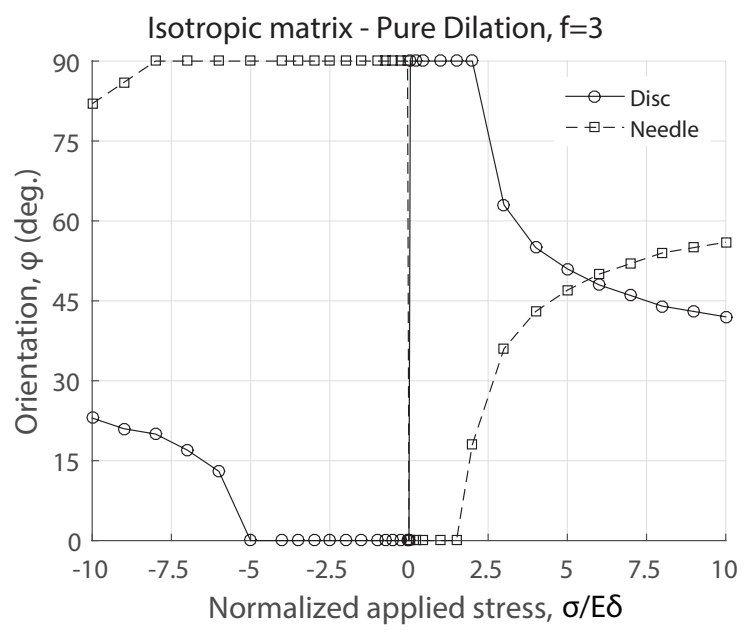

(d)

Figure 4: (a) Total potential energy $G^{*}$ as a function of precipitate's shape and applied uniaxial stress $\sigma_{33}^{o}$ for an undermatched case $(f=0.5)$ (b) Orientations of a disc and needle shaped precipitate corresponding to minimum potential energy for $f=0.5$. Similar results for an overmatched precipitate $(f=3)$ are shown in $(\mathrm{c})$ and $(\mathrm{d})$, respectively. Notations Disc-90 and Needle- $\|$ indicate that the disc is perpendicular and needle is parallel to the applied stress. An isotropic eigenstrain $\delta$ is used in the numerical calculations.

Numerical results of Pineau (1976) suggest that for an undermatched precipitate $(f=0.5)$ a tensile stress always favors a plate, whereas a compressive stress first favors a needle and then, at higher stresses, a plate shape again. Our numerical studies, however, predict that a disc shaped precipitate, under a uniaxial stress, is always the configuration of minimum potential energy. The difference in predictions between the two sets of numerical studies arises because the former is based on the assumption that a disc is always perpendicular to the applied stress whereas a needle is always aligned to it. Our numerical studies suggest that these simple orientations are not always the configurations of minimum energy. The orientation of a disc and needle shaped precipitate corresponding to minimum potential energy is presented in fig.4(b). For the case of a thin disc, there is a threshold tensile stress below which $\phi$ is zero, that is, the disc is 
perpendicular to the tensile stress. In uniaxial compression, a disc aligned with the stress axis $\left(\phi=90^{\circ}\right)$ leads to minimum energy until a threshold is reached. The threshold value, however, differs in tension and compression. Beyond this threshold stress the angle $\phi$ changes with the applied stress and at high magnitudes, the orientation of an elastic inhomogeneity is reached asymptotically (section 3.1). A similar behavior is observed for a needle shaped precipitate.

In contrast to an undermatched case, the effect of applied stress on the morphology of an over matched precipitate is more interesting. The total potential energy curve of a disc shaped precipitate is in the form of a cusp exhibiting a sharp discontinuity at the point of stress transition. A needle shaped precipitate also exhibits a similar behavior. In the absence of an external stress, a sphere was observed to be the most preferred shape for an overmatched precipitate, see fig.3. Except at low values of applied stress, a needle is the preferred configuration in tension and a thin disc in compression. The orientations corresponding to the minimum potential energy of a disc and needle shaped precipitate are shown in fig.4(d). In uniaxial tension, a disc aligned with the stress $\left(\phi=90^{\circ}\right)$ is the orientation for lower potential energy until a threshold stress is reached. Under compressive loading, a disc perpendicular to the applied stress leads to lower potential energy. At high values of applied stress, the orientation $(\phi)$ reaches a value that corresponds to the case of an elastic inhomogeneity, that is, $\phi=33.2^{\circ}$ for a disc and $65.76^{\circ}$ for a needle.

For completeness, numerical studies are extended to cover cases for very low and high values of elastic mismatch between the matrix and the precipitate $(f=0.1$ and 10$)$, see fig.5. For $f=1$, precipitate shape does not have any influence on the total potential energy (Eshelby, 1957).

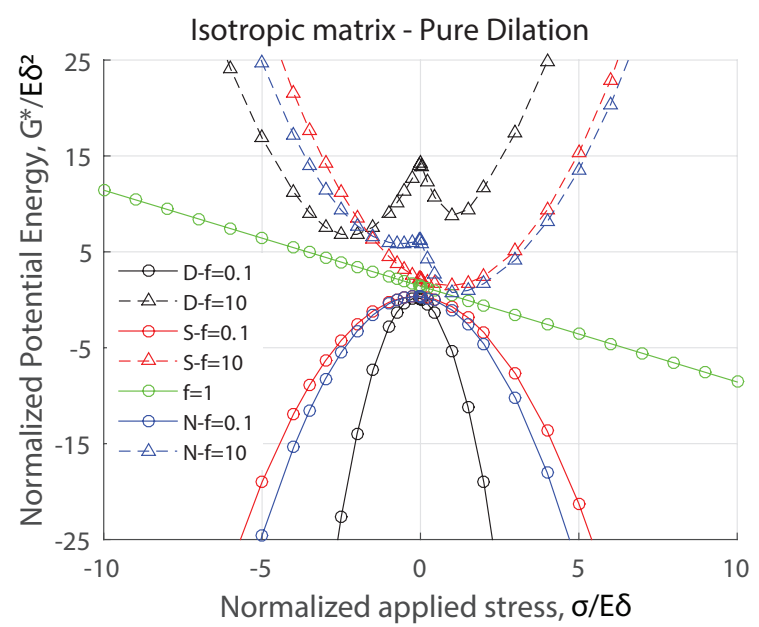

(a)

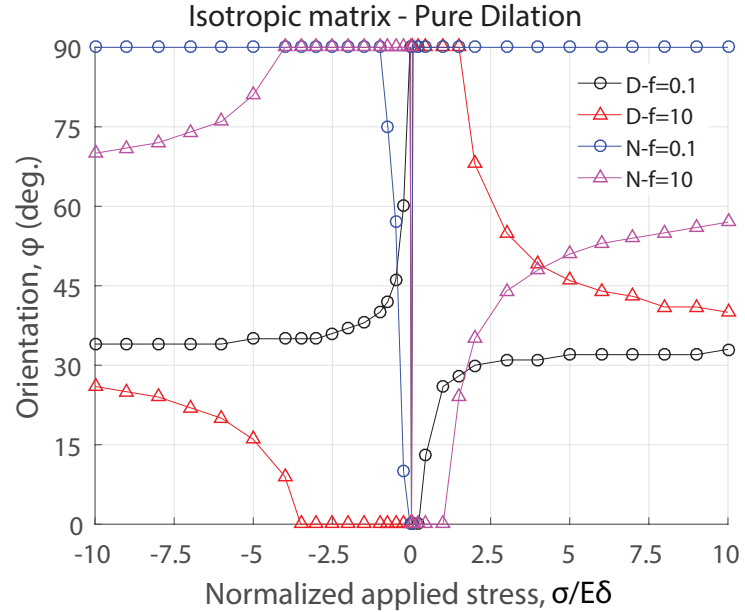

(b)

Figure 5: (a) Total potential energy $G^{*}$ as a function of precipitate shape and applied uniaxial stress $\sigma_{33}^{o}$ for different values of elastic mismatch factor $f(\mathrm{~b})$ Orientations of a disc and needle shaped precipitate corresponding to minimum potential energy. $D, S$ and $N$ refer to disc, sphere and needle, respectively. Eigenstrain $\delta$ is assumed to be purely dilatational.

We conclude this section by presenting some closed-form expressions for the orientation corresponding to minimum potential energy of a disc and a needle shaped precipitate, subjected to a uniaxial stress $\sigma_{33}^{o}$. These analytical expressions have provided insights and have developed further confidence in the results obtained from the numerical scheme (presented in section 2). 
For a disc shaped precipitate, the orientation angle $\phi$ that minimizes the total potential energy can be obtained from the following expression (section A.2 in Appendix),

$$
\sigma_{33}^{o}\left\{\sin ^{2} \phi-\frac{\nu}{1-\nu} \cos ^{2} \phi\right\}-\frac{\delta f E}{(f-1)(1-\nu)}=0
$$

For the case of an elastic inhomogeneity $(\delta=0)$, the above expression reduces to eq.(17). For an inhomogeneous inclusion, unlike the case of an elastic inhomogeneity, a threshold stress that is required to cause reorientation of a precipitate exists. It is of some interest to probe the reasons behind the existence of the threshold stress and examine the parameters that influence it. In the absence of an external stress, the strain energy of an inhomogeneous inclusion, in an isotropic elastic matrix, having dilatational eigenstrains is independent of precipitate orientation. As an external tensile stress is applied, the inhomogeneity strains in an undermatched precipitate $(f<1)$, being of opposite sign to the prescribed eigenstrains, reduces the equivalent eigenstrains. To be precise, $\varepsilon_{11}^{* *}$ and $\varepsilon_{22}^{* *}$ reduces and $\varepsilon_{33}^{* *}$ actually increases. As a result, the elastic stresses $\sigma_{11}$ and $\sigma_{22}$, inside the precipitate, resulting from the combined effect of eigenstrains and inhomogeneity, are reduced. The inhomogeneity strains are a function of precipitate orientation and so are the elastic stresses, see Appendix-A. The stresses inside the precipitate increase with angle $\phi$ and as a result, the inclusion energy (second term in the total potential energy $G^{*}$ in eq.(A-15)) increases. For an elastic inhomogeneity $\delta=0$, and the total potential energy scales with the applied stress, $\sigma_{33}^{o}$. The orientation corresponding to minimum potential energy, therefore, becomes independent of applied stress. For an inhomogeneous inclusion, at lower values of applied stress, it is this second term that dominates the total potential energy and, therefore, $\phi=0^{\circ}$ is the orientation corresponding to minimum potential energy for a disc shaped precipitate. For an overmatched case $(f>1)$, the inhomogeneity strains are of the same sign as the eigenstrains and the elastic stresses inside the precipitate decrease with angle $\phi$. Thus, at low values of applied stress, the orientation $\phi=90^{\circ}$ leads to minimum potential energy. As $\sigma_{33}^{o}$ increases, the inhomogeneity strains now start dominating over the prescribed eigenstrains. In this scenario, it is the elastic energy of an inhomogeneity, first term on the right hand side of eq.(A-15), that starts controlling the total potential energy. Therefore, in the limiting case of very high applied stress, the orientation corresponding to minimum potential energy of an inhomogeneity is reached.

For a disc shaped precipitate, the threshold stress that leads to its reorientation from $\phi=0^{\circ}$ can be obtained from the following expression,

$$
\left(\sigma_{33}^{o}\right)_{\mid \phi=0}=\frac{\delta f E}{(1-f) \nu}
$$

At very low values of $f$, the threshold stress tends to zero. As mentioned earlier, for $f=1$ there is no preferred orientation of a precipitate and the threshold stress, therefore, has no relevance. For an overmatched precipitate, a compressive stress is required to cause reorientation from $\phi=0^{\circ}$. At high values of elastic mismatch, $\sigma_{33}^{o} / E \delta \rightarrow-1 / \nu$. The threshold stress that is required to cause reorientation from $\phi=90^{\circ}$ can be calculated using the following equation,

$$
\left(\sigma_{33}^{o}\right)_{\mid \phi=90}=\frac{\delta f E}{(f-1)(1-\nu)}
$$

The orientation angle $\phi$ that minimizes the potential energy of a needle shaped precipitate can be obtained from (section A.3 in Appendix), 


$$
\sigma_{33}^{o}\left\{\bar{D} \sin ^{2} \phi+(1+f-2 \nu) \cos ^{2} \phi\right\}-\frac{\delta f E(1+f)}{(f-1)}=0
$$

Here, $\bar{D}$ is a complicated function of elastic mismatch factor $f$ and Poisson's ratio $\nu$, already defined in eq.(19). Similar to the case of a thin disc, corresponding expressions for the threshold stress of a needle shaped precipitate are,

$$
\begin{gathered}
\left(\sigma_{33}^{o}\right)_{\mid \phi=0}=\frac{\delta f E(1+f)}{(f-1)(1+f-2 \nu)} \\
\left(\sigma_{33}^{o}\right)_{\mid \phi=90}=\frac{\delta f E(1+f)}{(f-1) \bar{D}}
\end{gathered}
$$

It is perhaps worth mentioning that for certain magnitudes of applied stress $\sigma_{33}^{o}$, no real solution for $\phi$ that minimizes the total potential energy of a disc or a needle can be obtained analytically, see eqs.(20) and (23). For e.g., for a needle shaped precipitate with $f \leq 0.645$ no real solution for $\phi$ is obtained for $\sigma_{33}^{o} / E \delta \geq 0$. For such cases, the orientations corresponding to the minimum potential energy can be obtained numerically, as shown in figs. 4 and 5.

\section{Hydride precipitation in an elastic zirconium matrix}

We analyze here, the problem of hydride precipitation in zirconium. The $\alpha$-zirconium phase has a transversely isotropic response and its elastic constants were tabulated by Fisher and Renken (1964). Numerical values of the elastic moduli, used in this study, in Voigt notation are $C_{11}=$ $143.4 \mathrm{GPa}, C_{33}=164.8 \mathrm{GPa}, C_{12}=72.8 \mathrm{GPa}, C_{13}=65.3 \mathrm{GPa}, C_{44}=35.3 \mathrm{GPa}$ and $C_{66}=$ $32.0 \mathrm{GPa}$. The Cartesian axes $x_{1}$ and $x_{2}$, see fig.1, are in the basal plane of the matrix and $x_{3}$ is normal to it. The precipitated phase ( $\delta$-hydride) has a face-centred-cubic (FCC) structure. The anisotropy in its elastic properties is, however, not well-characterized. Leitch and Puls (1992) and Singh et al. (2008) have modeled it as isotropic elastic and the same assumption is invoked here. However, unlike the previous studies (Leitch and Puls, 1992; Singh et al., 2008), detailed analyses are carried out accounting for the elastic mismatch in the properties of $\delta$-hydride and $\alpha$-zirconium phases. For an isotropic elastic precipitate, the moduli $C_{11}^{*}$ and $C_{12}^{*}$ are sufficient to characterize its constitutive response. The elastic moduli of a hydride precipitate, used in our parametric studies, are obtained from the elastic constants of $\alpha$-zirconium using a linear scaling scheme,

$$
C_{11}^{*}=f C_{11}, \quad C_{12}^{*}=f C_{12}
$$

For a homogeneous case $(f=1), C_{11}^{*}=143.4 \mathrm{GPa}$ and $C_{12}^{*}=72.8 \mathrm{GPa}$. The corresponding values for the Young's modulus and Poisson's ratio of the hydride precipitate are $94.37 \mathrm{GPa}$ and 0.336 , respectively. These numerical values are almost the same as used in the study of Singh et al. (2008). A wide variation in the numerical values of elastic mismatch parameter $(0.1 \leq f \leq$ $10)$ is considered. Due to difference in the crystal structure of the matrix and the precipitate, the case $f=1$ does not mean identical elastic properties of the two phases. Precipitation of $\delta$-hydride in $\alpha$-zirconium is associated with a large volume change of about $17.2 \%$ (Carpenter, 1973). Transversely isotropic eigenstrains, that is, $\epsilon_{11}=\epsilon_{22}=0.0458$ and $\epsilon_{33}=0.072$ computed by Carpenter (1973) from lattice parameters are employed in our numerical calculations. 


\subsection{Effect of elastic mismatch on morphology and orientation of a hydride precipitate}

In this sub-section, the effect of mismatch in elastic constants between a hydride precipitate and zirconium matrix on the elastic strain energy is analyzed numerically. Four different shapes of precipitate namely; disc, sphere, needle and an oblate ellipsoid with aspect ratio $c / a=0.1$ are considered. Fig. 6 shows the plot of normalized strain energy $W^{*} / E \delta^{2}$ as a function of elastic mismatch factor, $f$. An average value of elastic modulus in the basal plane and in the transverse direction $\left(E_{11}+E_{33}\right) / 2$ ), and mean value of transformation strains $\delta=\varepsilon_{i i} / 3$ are used to normalize the elastic strain energy. The numerical values of $E$ and $\delta$ are $112.07 \mathrm{GPa}$ and 0.0545, respectively. The major axes of precipitates are assumed to lie in the basal plane. As it will be shown here, these orientations correspond to minimum strain energy.

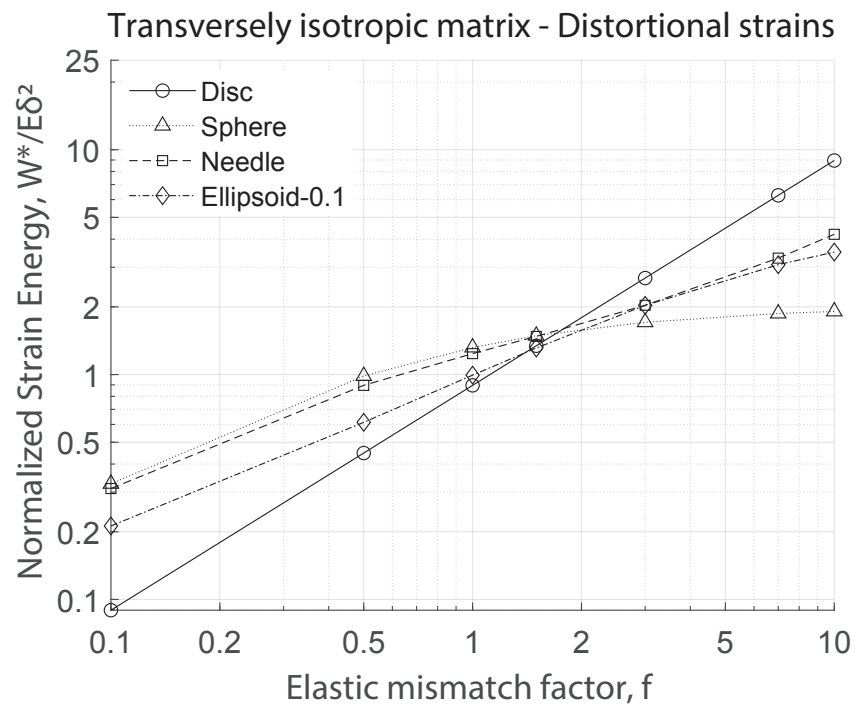

Figure 6: Effect of elastic mismatch between a hydride precipitate and zirconium matrix on the strain energy $W^{*}$. Four different shapes of precipitate namely disc, sphere, needle and an ellipsoid with aspect ratio $c / a=0.1$ are analyzed. Transversely isotropic eigenstrains $\epsilon_{11}=\epsilon_{22}=$ 0.0458 and $\epsilon_{33}=0.072$ as suggested by Carpenter (1973) are used in the numerical calculations

For a precipitate whose overall elastic properties are less or equal to that of the matrix $(f \leq 1)$, a thin disc leads to the minimum strain energy whereas a sphere corresponds to the highest strain energy. The difference in energies of spherical and needle shaped precipitates is, however, quite small. As expected, the strain energy for an ellipsoidal shape lies in between those of the disc and sphere. At values of $f$ around 1.5, a change in trend is observed. An ellipsoidal shaped precipitate having an aspect ratio $c / a=0.1$ leads to the minimum strain energy and the difference in energies for various shapes of precipitates also gets reduced. For $f \geq 2$, the trend is completely reversed; a spherical shape then leads to minimum strain energy and a thin disc corresponds to highest strain energy. A similar effect of precipitate morphology on elastic strain energy was observed by Mori et al. (1978) for a FCC copper matrix.

We now analyze the effect of precipitate orientation, for a given shape, on the elastic strain 
energy. Numerical calculations are carried out for two different values of elastic mismatch $(f$ $=0.5$ and 1.5) and the results are summarized in fig.7. Since the matrix elastic response and the eigenstrains arising from hydride precipitation (Carpenter, 1973) are transversely isotropic, angle $\theta$ shown in fig.1, has no relevance. The orientation of an oblate spheroid is identified by the normal to the broad face whereas that of a prolate spheroid by the long axis. Thus, $\phi=0^{\circ}$, means that a disc or an oblate ellipsoid lies in the basal plane, whereas a needle is oriented in the transverse direction. For the case $f=0.5$, a disc shaped precipitate lying in the basal plane leads to minimum strain energy, whereas a needle oriented along the c-direction (transverse) results in the highest strain energy. For increasing values of angle $\phi$, the disc and ellipsoidal shapes lead to a continuous rise in the elastic strain energy, whereas a needle shaped precipitate shows the reverse trend. The strain energy for the latter reaches a minimum when it lies in the basal plane, although the numerical value is much higher than that of a disc lying in the basal plane. For a spherical precipitate, as expected, the strain energy is independent of orientation. Except for the case of a needle oriented almost normal to the basal plane $(\phi \leq 14)$, a spherical shape leads to the highest strain energy.

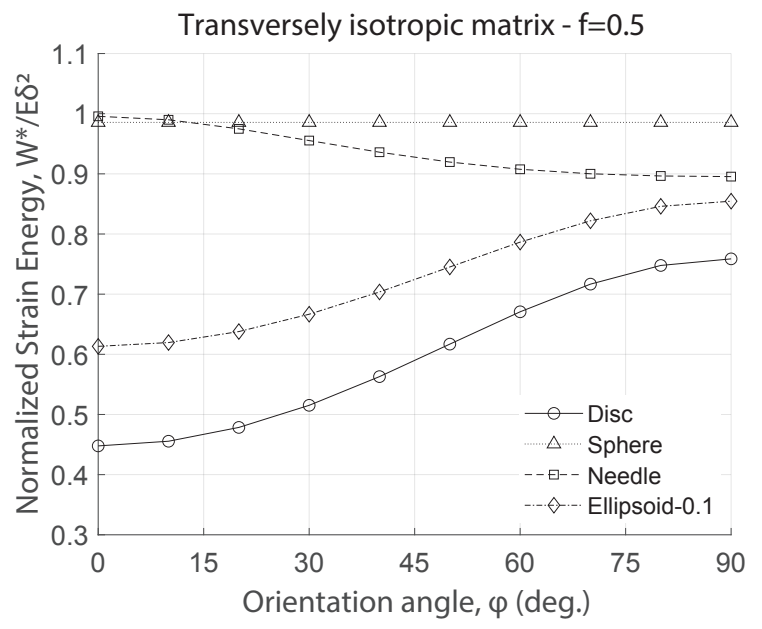

(a)

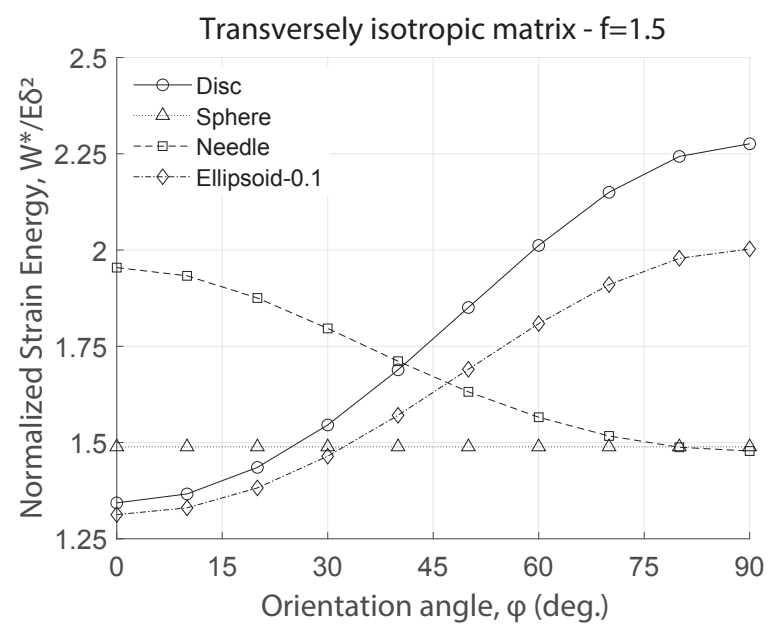

(b)

Figure 7: Elastic strain energy $W^{*}$ for different shapes of precipitate as a function of orientation angle $\phi$. Numerical results for two different values of elastic mismatch factor $f=0.5$ and 1.5 are shown in (a) and (b), respectively. Eigenstrains $\epsilon_{11}=\epsilon_{22}=0.0458$ and $\epsilon_{33}=0.072$ are used in the numerical calculations

For the case $f=1.5$, an oblate ellipsoid with an aspect ratio $c / a=0.1$, lying in the basal plane, leads to minimum strain energy, although it is not much different from that of a thin disc. For an isotropic elastic matrix, Kato et al. (1996) also observed a similar behavior. These authors reported that when a precipitate is stiffer than the surrounding matrix, the shape corresponding to minimum strain energy may not be a disc, sphere or needle but some intermediate oblate or prolate spheroid. It can be observed from fig.7(b) that for all angular orientations, the strain energy of an ellipsoidal precipitate, with aspect ratio $c / a=0.1$, remains lower than that of a disc. Similar to an undermatched case, the strain energy of a needle attains its minimum value when it is lying in the basal plane. A disc lying normal to the basal plane $\left(\phi=90^{\circ}\right)$ leads to 
highest strain energy. The tendency for a spherical precipitate to become the lowest energy configuration at higher values of $f$ is clearly visible.

\subsection{Role of external stress on the mechanics of hydride reorientation}

We discuss here, the effect of an applied uniaxial stress on the orientation of a zirconium hydride precipitate. The potential energy of a body in the absence of a precipitate $G_{o}$ is just a constant for a given configuration. Thus, the role of an external stress on the mechanics of hydride reorientation can be analyzed by examining the change in Gibbs free energy $\left(G^{*}\right)$ as a function of precipitate orientation for a given shape and magnitude of applied stress. Since the zirconium matrix is transversely isotropic, the effect of stress direction with respect to matrix crystallographic planes is accounted for. Two limiting cases namely; an external stress applied along the basal plane $\sigma_{11}^{o}$ and in the transverse direction $\sigma_{33}^{o}$ are analyzed.

Plots of normalized potential energy $G^{*} / E \delta^{2}$ as a function of applied stress for an undermatched precipitate $(f=0.5)$ are presented in fig.8(a). Only disc and needle shaped precipitates are analyzed. For different magnitudes of applied tensile stress acting in the transverse direction, a disc shaped precipitate leads to minimum potential energy. In the absence of an external stress, as observed in fig.7(a), the orientation $\phi=0^{\circ}$, that is, a disc lying in the basal plane leads to minimum strain energy. As the magnitude of external tensile stress acting in the transverse direction is increased, the angle $\phi$ gradually increases and the behavior is similar to that of an isotropic elastic matrix. For the case of an external stress acting along the basal plane, an interesting deviation is noted. Once the tensile stress $\sigma_{11}^{o}$ reaches some threshold value, the orientation of a disc shaped precipitate suddenly changes to $\phi=90^{\circ}$. Based on elastic calculations, the threshold tensile stress is estimated to be around 1.65 GPa. With a further increase in the applied stress, a reorientation phenomenon can be observed again. The case of a uniaxial compressive stress acting in the transverse direction also exhibits a similar behavior. Once $\sigma_{33}^{o}$ exceeds $-1.46 \mathrm{GPa}$, the orientation flips from $\phi=0^{\circ}$ to $\phi=90^{\circ}$. Thus, unlike the case of an isotropic matrix, a complete reversal of orientation at the point where stress makes a transition from tension to compression or vice-versa is not observed. This is essentially a consequence of anisotropy in the elastic properties of zirconium and the anisotropic transformation strains resulting from hydride precipitation. The second reorientation phenomenon, observed at higher magnitudes of applied stress is a consequence of the inhomogeneity. Due to anisotropy in the elastic constants of the zirconium matrix, the orientation of a disc shaped elastic homogeneity depends on the mismatch factor $f$, though the dependence is quite weak. A needle shaped precipitate also exhibits a similar behavior.

It is interesting to note that the predicted threshold tensile/compressive stress (based on elastic analysis) for a complete reorientation of a single disc shaped hydride precipitate is in agreement with the numerical results obtained from the phase field theory. Based on 2D elastic phase field simulations, Bair and Zaeem (2017) reported that a tensile strain of 0.021 acting along the basal plane, corresponding to an elastic stress of $1.78 \mathrm{GPa}$, causes a complete reorientation of a single nanoscale hydride precipitate. Similarly, a compressive strain of 0.018 acting in the transverse direction, corresponding elastic stress of $-1.53 \mathrm{GPa}$, was shown to lead to hydride reorientation. More detailed studies employing 3D phase field simulations (see Motta et al., 2019) led to a somewhat lower value of $894 \mathrm{MPa}$ as the threshold tensile stress for reorientation of a single hydride platelet. 


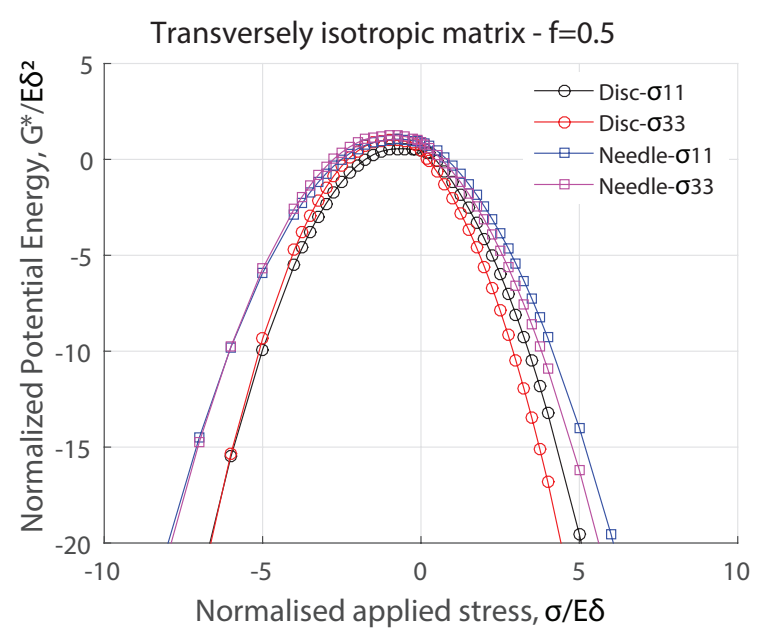

(a)

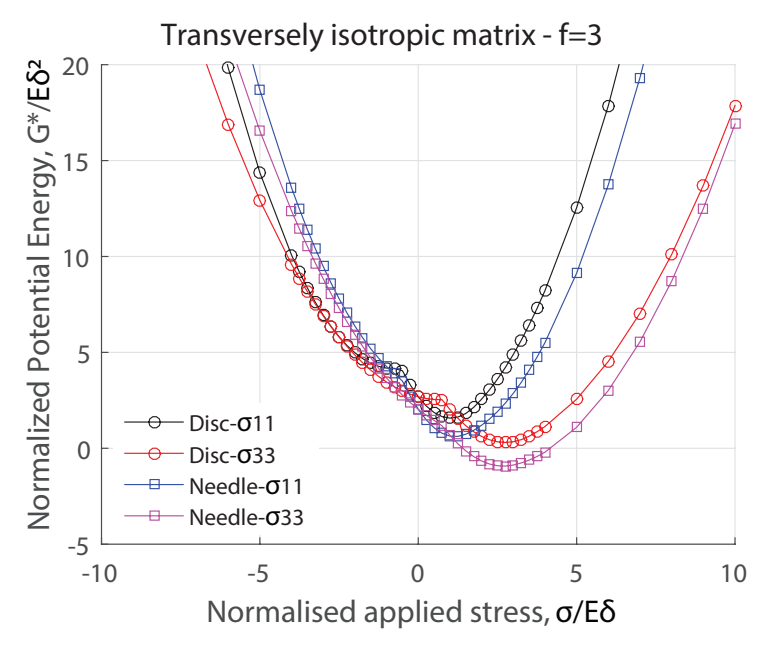

(c)

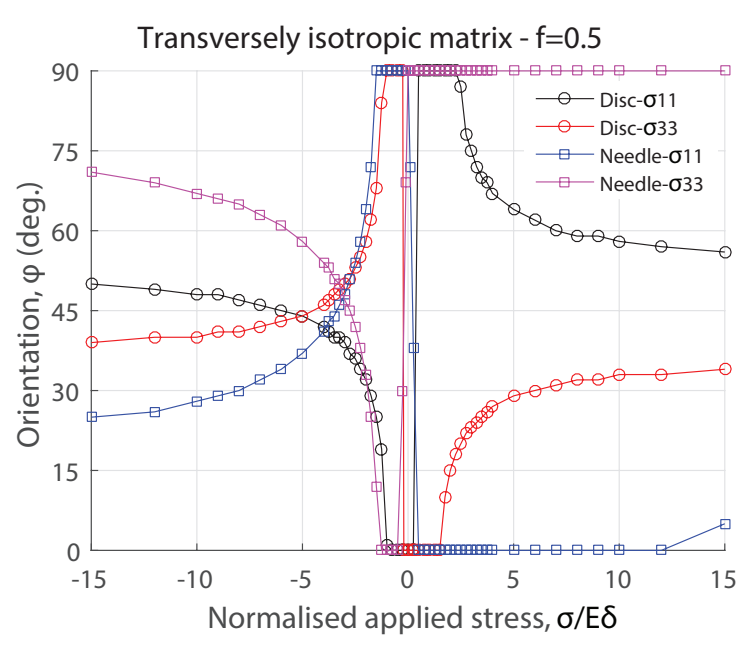

(b)

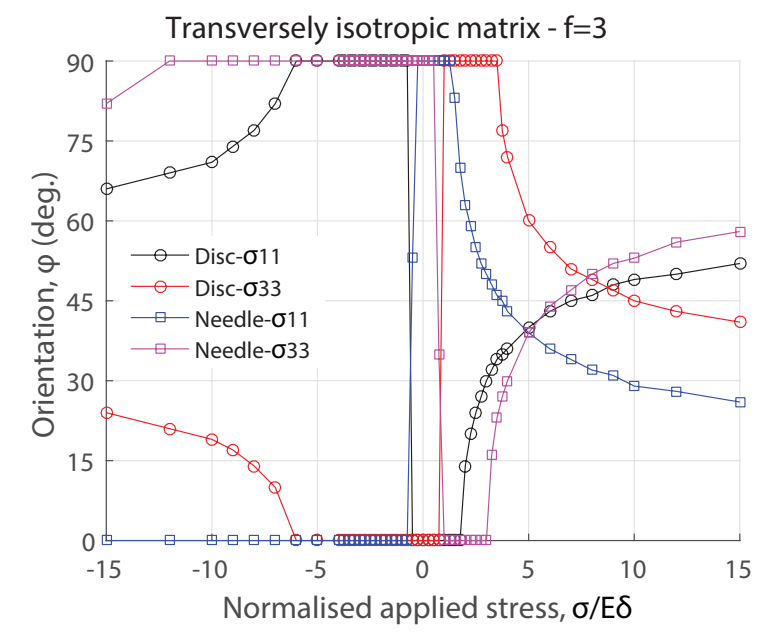

(d)

Figure 8: (a) Total potential energy $G^{*}$ of a disc and needle shaped precipitate as a function of applied stress for an undermatched case $(f=0.5)$. Notations $\sigma_{11}^{o}$ and $\sigma_{33}^{o}$ correspond to applied stress acting along the basal plane and in the transverse direction, respectively. (b) Orientation of a disc and needle shaped precipitate corresponding to minimum potential energy for $f=0.5$. Similar results for an overmatched precipitate $(f=3)$ are shown in (c) and (d), respectively. Eigenstrains $\epsilon_{11}=\epsilon_{22}=0.0458$ and $\epsilon_{33}=0.072$ are used in the numerical calculations

Unlike an undermatched case, the applied stress has a noticeable influence on the morphology of an overmatched precipitate. A sphere is observed to be the most preferred shape for $f=3$, see fig.6. Except at very low magnitudes of applied stress, a needle is observed to be the preferred configuration in tension and a thin disc under external compression. The effect of matrix anisotropy is quite visible in the potential energy plots of an overmatched precipitate. The discontinuity in slope of the potential energy curves for disc and needle shaped precipitates does not correspond to the stress reversal point. The discontinuity is observed in the tensile stress regime for $\sigma_{33}^{o}$ and in the compressive regime for $\sigma_{11}^{o}$. This behavior is also reflected in the orientation plots shown in fig.8(d). Similar to the undermatched case, a threshold stress 
leading to a sudden change in the precipitate's orientation followed by a gradual reorientation phenomenon at higher stress is observed for both disc and needle shaped precipitates.

\subsection{Stress biaxiality effect on hydride reorientation}

In this sub-section, the effect of applied stress biaxiality on the orientation of disc and needle shaped precipitates is analyzed numerically. As before, two limiting cases corresponding to an external stress acting in the transverse direction and along the basal plane of the zirconium matrix are considered. Over and above this major external stress, a minor stress is applied orthogonally in the $x_{1}-x_{3}$ plane. Stress biaxiality is characterized by a parameter $\beta$ defined as the ratio of minor to major external stress.

Orientation of a disc shaped precipitate, subjected to an applied biaxial stress, is shown in fig.9. For direct comparison, results for the case of uniaxial stress, $\beta=0$, are also included. In the absence of external stress, the orientation $\phi=0^{\circ}$ leads to minimum strain energy. At magnitudes of uniaxial tensile stress, higher than the threshold limit, the tendency for an undermatched precipitate is to attain an orientation that is normal to the applied stress whereas an overmatched precipitate prefers a tangential orientation, see fig.8. For the case of uniaxial compression, the exact opposite behavior is observed. A further increase in applied stress, leads to a gradual reorientation of a thin disc. Application of a minor stress in the orthogonal direction results in an increase in the threshold stress although it is not clearly visible at the scale used in fig.9(a) and (b). Since the biaxial state reduces the shear stress inside a precipitate, the gradual reorientation phenomenon is also shifted towards higher stress magnitudes. Moreover, as stress biaxiality increases a precipitate prefers to attain an orientation that is more towards the normal to the major applied stress.

To gain further insights on the role of elastic mismatch and stress biaxiality in the mechanics of hydride reorientation it is useful to evaluate the response analytically. A complete theoretical treatment of an inhomogeneous inclusion in an anisotropic matrix is quite involved. We therefore, analyze the simplified case of an elastic inhomogeneity under biaxial stress in an isotropic matrix, see Appendix-B. Closed-form expressions are developed to analyze the influence of mismatch factor $f$ and biaxiality parameter $\beta$ on the preferred orientation of a precipitate. For a disc shaped inhomogeneity in an isotropic elastic matrix under bi-axial stress, the orientation minimizing the potential energy can be obtained from the following equation (Appendix-B),

$$
\tan \phi=\sqrt{\frac{\nu-(1-\nu) \beta}{1-\nu-\nu \beta}}
$$

For a typical value of $\nu=0.3$, the angle $\phi=33.2^{\circ}, 24.1^{\circ}$ and $3.8^{\circ}$ corresponding to $\beta=0,0.25$, 0.425 , respectively. For $0.43 \leq \beta<1$ an analytical solution for $\phi$ does not exist. Numerical solutions, however, suggest that for this range of stress biaxiality, a disc perpendicular to the major applied stress, $\phi=0^{\circ}$, is the configuration of minimum potential energy. A disc shaped inhomogeneity lying in an anisotropic elastic matrix also exhibits a similar behavior. The plots shown in fig.9 depict the orientation corresponding to minimum potential energy of an inhomogeneous inclusion lying in an anisotropic elastic matrix. At very high magnitudes of applied stress, the effect of transformation strain decays, thus, leading to the orientation attained by an elastic inhomogeneity. When the major external stress acts in the transverse direction of the zirconium matrix, our numerical calculations predict that the interaction energy 
is minimum when $\phi=36^{\circ}, 31^{\circ}, 21^{\circ}$ and $0^{\circ}$ corresponding to $\beta=0,0.25,0.5$ and 1 , respectively. These are, therefore, the limiting orientations which the curves shown in fig.9(a) attain at very high magnitudes of applied stress (outside the range of external stress shown in the figure). Corresponding orientations for the case where the major external stress acts along the basal plane are $\phi=53^{\circ}, 60^{\circ}, 90^{\circ}$ and $0^{\circ}$.

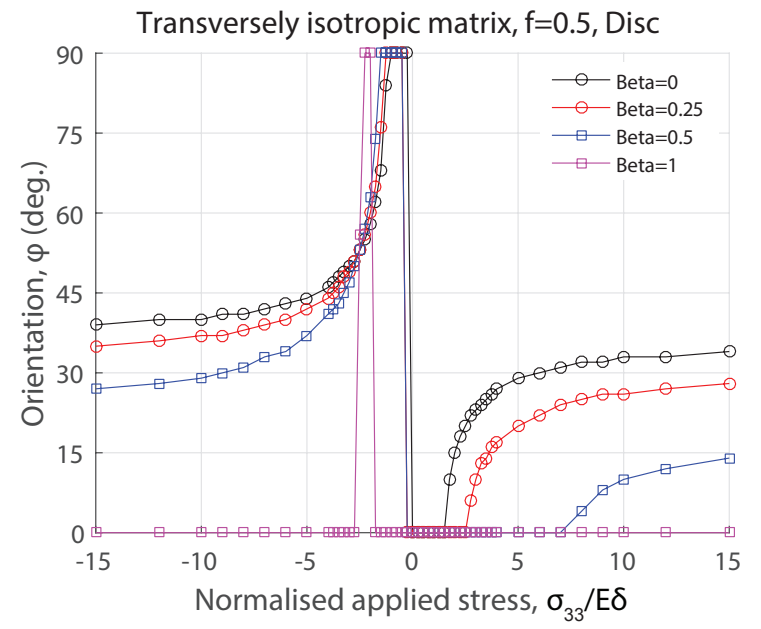

(a)

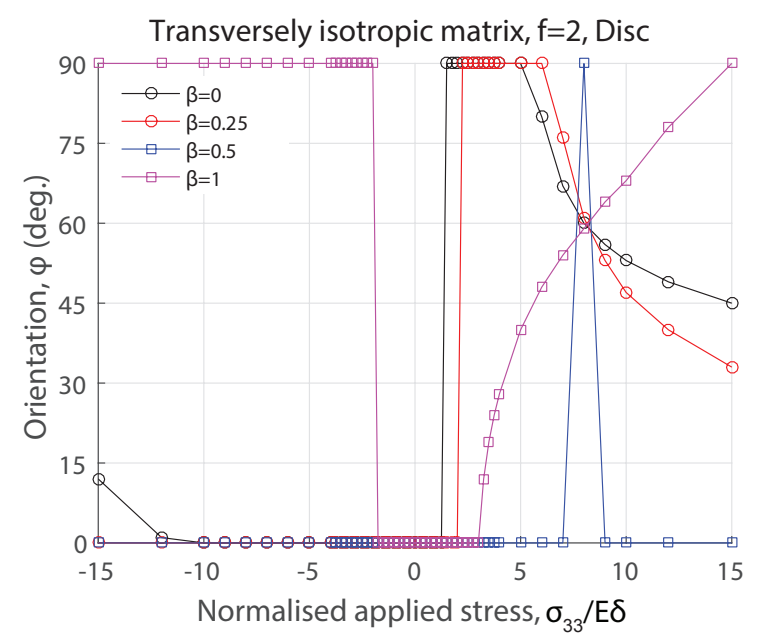

(c)

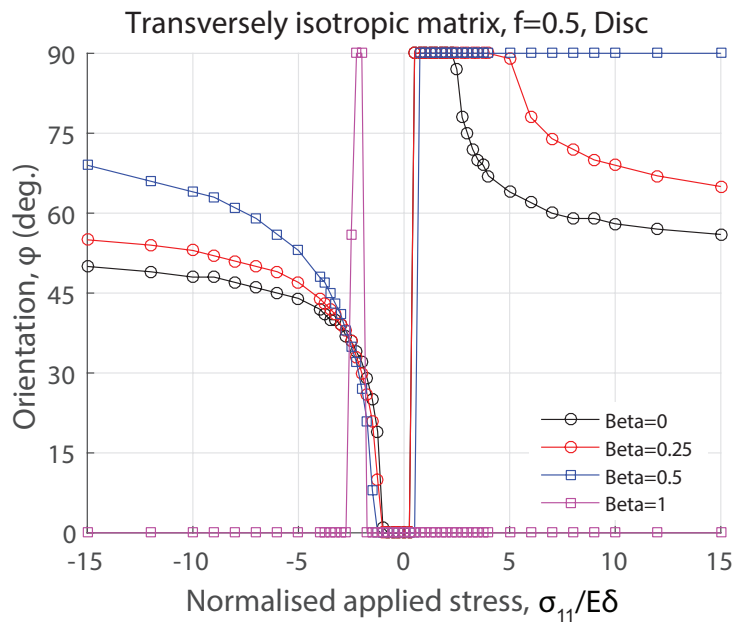

(b)

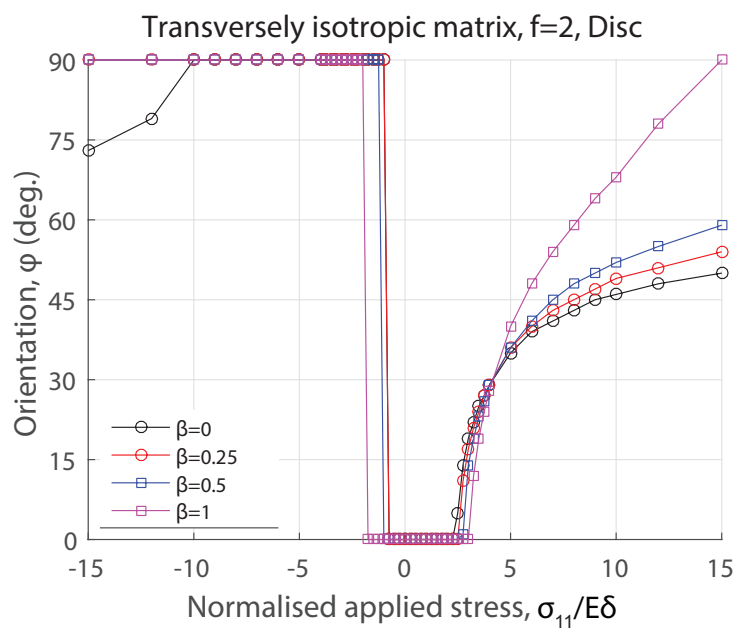

(d)

Figure 9: Orientation of a disc shaped precipitate corresponding to minimum potential energy $G^{*}$ as a function of applied stress for an undermatched case $(f=0.5)$. Parameter $\beta$ characterizes the stress biaxiality. The major external stress is assumed to act (a) in the transverse direction and (b) along the basal plane. Corresponding plots for an overmatched precipitate $(f=2)$ are shown in (c) and (d), respectively. Eigenstrains $\epsilon_{11}=\epsilon_{22}=0.0458$ and $\epsilon_{33}=0.072$ are used in the numerical calculations

A sharp narrow peak observed for the case of an undermatched disc shaped precipitate subjected to equi-biaxial stress $(\beta=1)$, see fig.9(a), is a consequence of anisotropy in the transformation strains. In the absence of applied shear stress, a precipitate lying in the basal plane 
minimizes the interaction energy of inhomogeneity. Therefore, at high magnitude of applied stress, where the effect of transformation strains become insignificant, the orientation $\phi=0^{\circ}$ leads to minimum potential energy. This is irrespective of applied loading being tensile or compressive. At lower magnitude of applied stress, the inhomogeneity strains are, however, dominated by the phase transformation strains. For the case of tensile loading, the inhomogeneity strains leads to minimum interaction energy at $\phi=0^{\circ}$. The in-plane components of the equivalent eigenstrains are such that the elastic stresses inside the precipitate and, hence, the inclusion energy is also minimum in the basal plane. Therefore, for applied tensile stresses, the total potential energy of a hydride precipitate is observed to always be minimum at $\phi=0^{\circ}$. The same is not true for compressive loading. At lower stress levels, the inhomogeneity strains lead to minimum interaction energy at $\phi=90^{\circ}$. Thus, the variation of interaction energy as a function of precipitate orientation exhibits an opposite trend to that of the inclusion energy. Their relative contribution to the total potential energy dictates the orientation of the precipitate. At some stress levels, the orientation $\phi=90^{\circ}$ minimizes the total potential energy. Once the magnitude of applied stress is high, the effect of transformation strains diminishes and the interaction energy becomes minimum at $\phi=0^{\circ}$. Consequently, the orientation leading to minimum potential energy rapidly decreases from $90^{\circ}$ to $0^{\circ}$ and remains the same thereafter.

For an overmatched case, the role of interaction energy resulting from shear stress in determining a precipitate's orientation is limited. To be precise, the shear strain generated in an overmatched precipitate, for a given applied stress, is of much smaller magnitude than that of an undermatched case. This is consistent with the results of an isotropic matrix, see fig.2. When a uniaxial stress is applied in the transverse direction, see fig.9(c), the interaction energy is observed to be minimum at $\phi=30^{\circ}, 0^{\circ}, 0^{\circ}$ and $90^{\circ}$ corresponding to $\beta=0,0.25,0.5$ and 1 , respectively. Similar to an undermatched case, a sharp peak in the orientation plot is also observed for an overmatched precipitate. Since the inhomogeneity strains in the two cases are of opposite sign, the peak is observed in the tensile stress regime. No such sharp peak is observed when the major external stress acts along the basal plane. For this case, fig.9(d), the interaction energy is observed to be minimum when $\phi=60^{\circ}, 66^{\circ}, 78^{\circ}$ and $90^{\circ}$ corresponding to $\beta=0,0.25,0.5$ and 1 , respectively. At high magnitude of applied stress, these are essentially the orientations that corresponds to minimum potential energy.

Numerical results of a needle shaped precipitate are shown in fig.10. For an undermatched case $(f=0.5)$ subjected to a uniaxial stress $(\beta=0)$ acting in the transverse direction, see fig.10(a), the interaction energy of the inhomogeneity is observed to be minimum at $\phi=79^{\circ}$. Due to asymmetry in the tensile and compressive stress regimes, the indication towards attaining this orientation is not visible for the range of tensile stress shown in fig.10(a). The corresponding value for the case of external stress acting along the basal plane, fig.10(b), is $17^{\circ}$. For the case of biaxial stress, interaction energy is minimum when the needle is normal to the major applied stress, that is, $\phi=90^{\circ}$ or $0^{\circ}$. In the absence of external stress, a needle lying in the basal plane $\left(\phi=90^{\circ}\right)$ is the preferred orientation. In between these two extremes, the relative contribution of interaction and the inclusion energy dictates the orientation that leads to minimum potential energy. Similar to the case of a thin disc, a sharp peak in the orientation plot is observed for the case of equi-biaxial stress, see fig.10(a). The underlying reasons are similar to those discussed earlier. The interaction energy for an overmatched case $(f=2)$, under a uniaxial stress acting in the transverse direction, is minimum at $\phi=74^{\circ}$, see fig.10(c). When the external stress acts along the basal plane, fig.10(d), the corresponding value is $1.5^{\circ}$. For a biaxial state of stress, the orientation $\phi=0^{\circ}$ or $90^{\circ}$ minimizes the interaction energy. An overmatched precipitate exhibits 
a sharp peak in the orientation response for $\beta=0.5$, see fig.10(c). Analogous to the case of a thin disc, the peak is observed in the tensile stress regime. For the case of external stress acting along the basal plane, no such peak is observed.

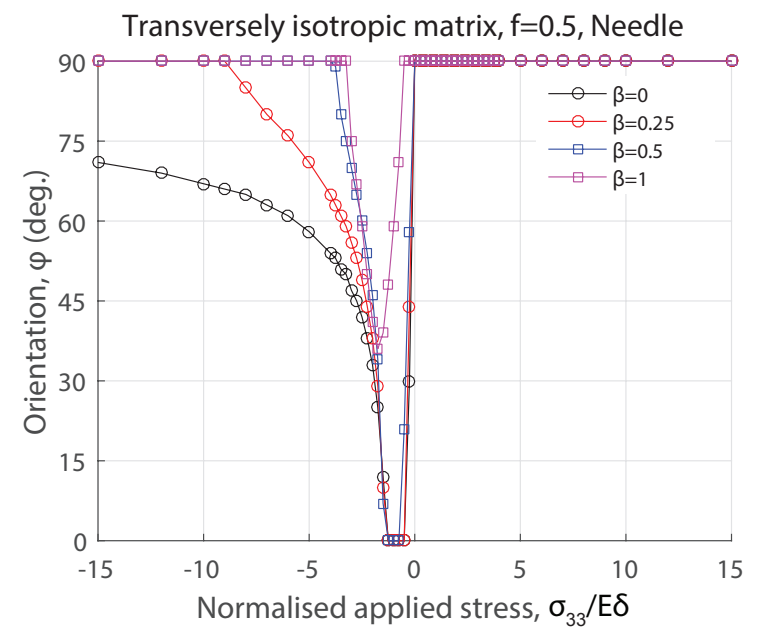

(a)

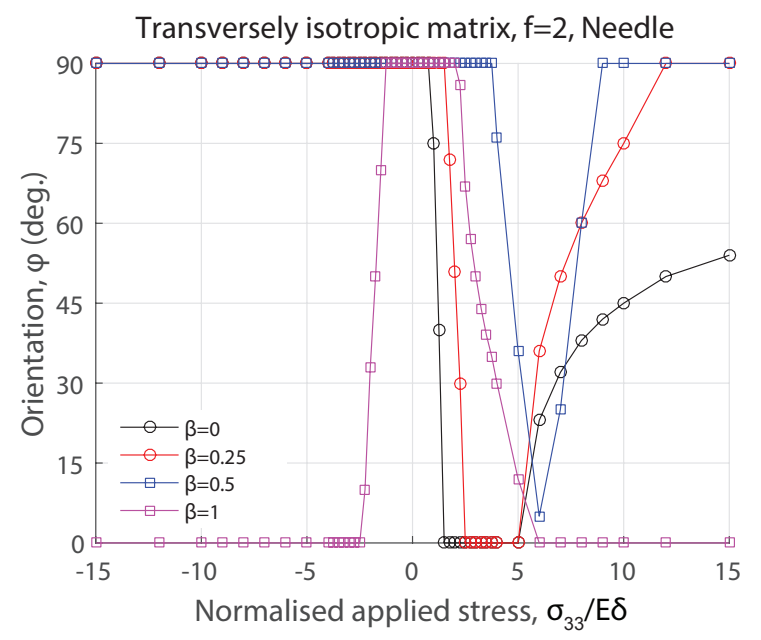

(c)

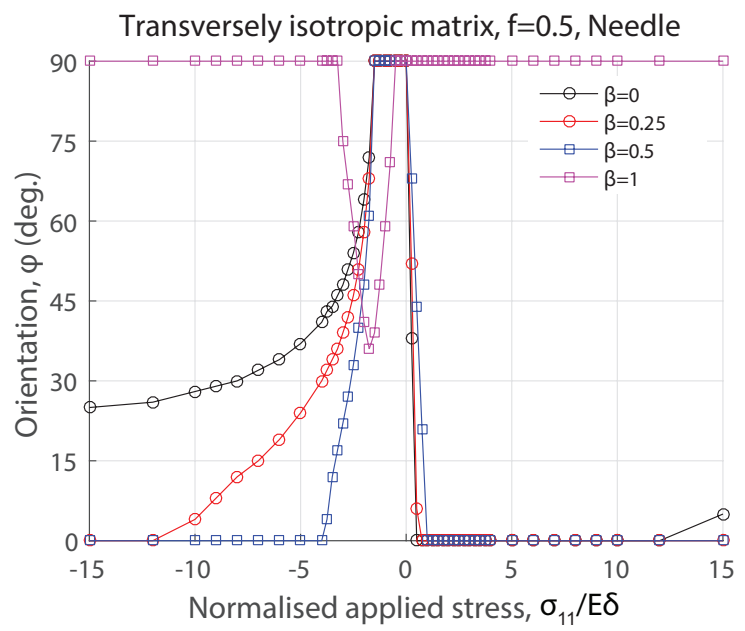

(b)

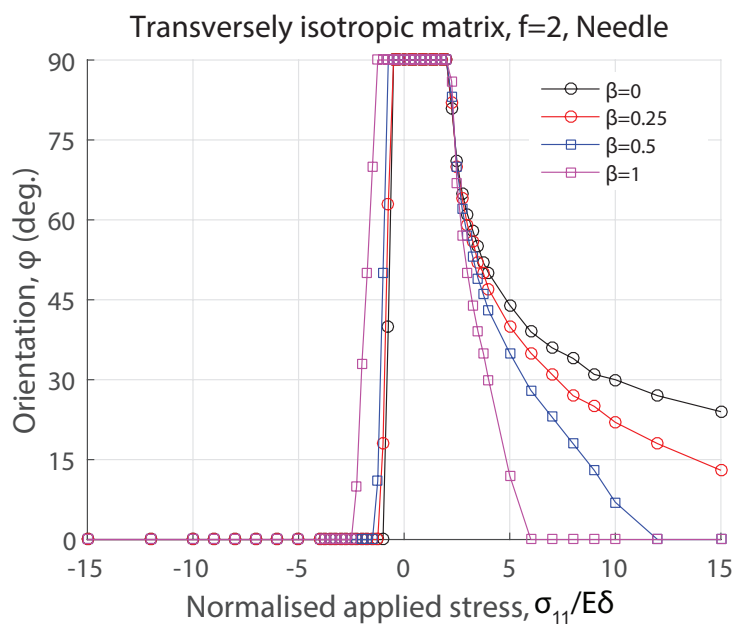

(d)

Figure 10: Orientation of a needle shaped precipitate corresponding to minimum potential energy $G^{*}$ as a function of applied stress for an undermatched case $(f=0.5)$. Parameter $\beta$ characterizes the stress biaxiality. The major external stress is assumed to act (a) in the transverse direction and (b) along the basal plane. Corresponding plots for an overmatched precipitate $(f=2)$ are shown in $(\mathrm{c})$ and $(\mathrm{d})$, respectively. Eigenstrains $\epsilon_{11}=\epsilon_{22}=0.0458$ and $\epsilon_{33}=0.072$ are used in the numerical calculations

\subsection{Effect of anisotropic interfacial energy on orientation of a disc shaped hydride precipitate}

The results presented in the previous sub-sections are based on the assumption that the hydride precipitate is sufficiently large for the contribution of interfacial energy to the energetics of 
hydride precipitation to be safely ignored. A number of theoretical studies, however, suggest that the preferential orientation of hydride precipitates under stress is most effective during the nucleation stage of precipitation (Ells, 1970; Massih and Jernkvist, 2009). The interfacial energy constitutes a substantial fraction of the overall driving force causing the nucleation of a precipitate. Massih and Jernkvist (2009) have suggested a strong anisotropy in the interfacial energy between a $\delta$-hydride precipitate and the $\alpha$-zirconium matrix. The surface energies along the $a$ and $c$-axis of the zirconium matrix were reported as 0.065 and $0.28 \mathrm{~J} / \mathrm{m}^{2}$, respectively. In a recent work, Han et al. (2019) computed the interfacial energies of a $\delta$-hydride precipitate using the density functional theory. Their computational predictions suggest that $\gamma_{\| a}$ and $\gamma_{\| c}$ are 0.053 and $0.15 \mathrm{~J} / \mathrm{m}^{2}$, respectively.

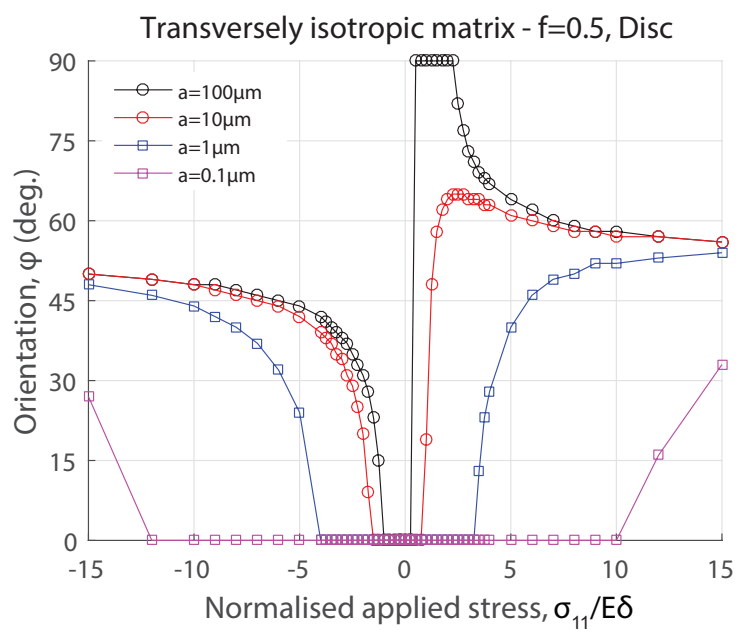

(a)

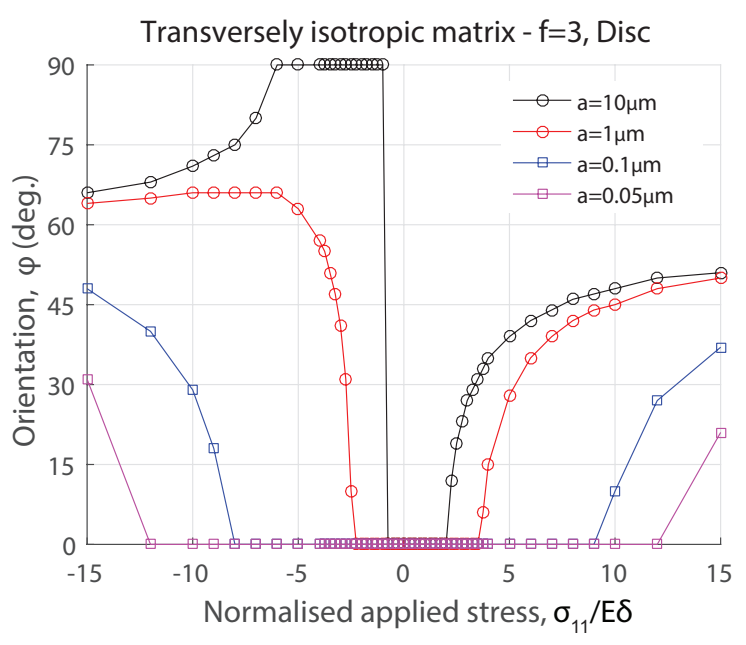

(b)

Figure 11: Effect of anisotropic interfacial energy on the orientation of a disc shaped precipitate corresponding to minimum Gibbs free energy $G^{*}$. The external stress is acting along the basal plane. Numerical results for two different values of elastic mismatch factor $f=0.5$ and 3 are shown in (a) and (b), respectively. Parameter $a$ is the length of semi-major axis of the precipitate. Eigenstrain $\epsilon_{11}=\epsilon_{22}=0.0458$ and $\epsilon_{33}=0.072$ are used in the numerical calculations

We now proceed to examine the effect of anisotropic interfacial energy on the orientation of a disc shaped $\delta$ - hydride precipitate subjected to an external stress. Incorporation of interfacial energy in the calculation of overall driving force (Gibbs free energy $G^{*}$ ) introduces a length scale in the computational model. The orientation of a hydride precipitate subjected to an external stress applied along the basal plane $\sigma_{11}^{o}$ is shown in fig.11. As before, numerical results are presented for two different values of elastic mismatch factor, that is, $f=0.5$ and 3. The anisotropic interfacial energies suggested by Massih and Jernkvist (2009) are used in our calculations. The orientation dependence of the interfacial energy $\gamma(\phi)$ modeled in our analysis is similar to that obtained from a quadratic function, (see Han et al., 2019). The long side of a disc shaped precipitate, $a$, is chosen to represent the length scale in our numerical results. The material properties of the $\delta$-hydride and $\alpha$-zirconium matrix and the anisotropic eigenstrains used in our calculations have already been described earlier, see section 4 . For an undermatched case $(f=0.5)$, the effect of interfacial energy on the orientation of a disc shaped 
precipitate becomes insignificant provided $a \geq 100 \mu \mathrm{m}$. For this size of hydride, the interfacial energy is very small and as a result, a precipitate attains an orientation that is controlled by the total potential energy. As the size of hydride reduces, the volume of precipitate decreases in much higher proportion as compared to the surface area with the consequence that interfacial energy becomes a significant fraction of the Gibbs free energy $G^{*}$. For values of $a \leq 0.1 \mu \mathrm{m}$, the interfacial energy dominates over the potential energy and a hydride precipitate exhibits a tendency to remain in the basal plane. An overmatched precipitate $f=0.3$ also shows a similar behavior.

\section{An assessment of loading path on the energetics and dissipation in an elastic-plastic precipitate}

The mechanism of precipitation is typically influenced by the coherent stresses resulting from a phase transformation. The presence of an external stress during precipitation leads to interaction energy which influences the thermodynamics of the system. Some effects of external stress on the morphology and orientation of precipitates have already been examined in Sections 3 and 4. For linear elastic problems, the sequence of application of an external stress relative to phase transformation has no relevance as the final state of stress and strains are independent of loading path. Unfortunately, this is not the case for problems involving plastic deformations. The precipitation of $\delta$-hydride in an $\alpha$-Zr matrix is associated with a large lattice dilatation (Carpenter, 1973). The crystallographic coherence between a hydride precipitate and zirconium matrix is fairly high and dislocations are necessary to accommodate the lattice misfit. TEM studies of Bai et al. (1994) show a much higher dislocation density inside the hydrides, thus, suggesting a self-accommodation of the phase transformation strains within the precipitate. The dislocations localized in the vicinity of hydrides lead to plastic deformations in the matrix. Numerical results of Leitch and Puls (1992), employing phenomenological plasticity (isotropic $J_{2}$ flow theory), indicate that for flat oblate shaped inclusions the main cause of reduction in accommodation energy is the plastic relaxation in the hydride. Due to a high stress triaxiality in the matrix, only limited plastic relaxation occurs there. Another important consideration in the plastic relaxation of the accommodation energy is the yield strength mismatch between the hydride precipitate and the zirconium matrix. The experimental results suggest that for the range of service temperatures typically experienced by the pressure tubes used in CANDU-type reactors, the yield strength of $\delta$-hydride is significantly less than that of the $\alpha$-zirconium matrix. Using the available experimental data, Sharma et al. (2018) have carried out numerical calculations to determine the preferred shape and orientation of the hydrides at different service temperatures by minimizing the accommodation energy. Similar to the findings reported by Leitch and Puls (1992), the numerical results of Sharma et al. (2018) also show that for a thin disc shaped precipitate, the plastic relaxation in the hydride is mainly responsible for the reduction in accommodation energy.

As the above-mentioned studies suggest, a detailed assessment of plasticity on the energetics and dissipation associated with hydride precipitation may require a full nonlinear elastic-plastic or crystal plasticity based formulation. It is, however, worth examining whether an approximate assessment of such effects can be carried out. We, therefore, develop a numerical scheme to assess the influence of loading path on the Gibbs free energy and plastic dissipation in an 
elastic-plastic precipitate lying in a transversely isotropic elastic matrix. The proposed scheme is then utilized to examine the effect of the precipitate's plasticity on the phenomenon of stress reorientation of zirconium hydrides. The validity of our proposed simplified scheme is then assessed in a latter section where an account of precipitate and matrix plasticity is made using plane strain finite element analyses, see section 7.

The numerical scheme developed in section 2 can be utilized with some modifications to analyze the problem of an elastic-plastic precipitate lying in an elastic matrix. Since Eshelby's tensor components $S_{i j m n}$ are utilized to compute the stress and strain fields inside a plastically deforming precipitate, our proposed numerical scheme is based on a small-strain formulation. An incremental approach is adopted and an increment in prescribed/effective transformation strains within the precipitate can be specified in terms of increment in actual eigenstrains and the plastic strain increment,

$$
d \varepsilon_{i j}^{P r}=d \varepsilon_{i j}^{T}+d \varepsilon_{i j}^{p}
$$

Invoking the assumption of small-strain deformation, the total strain within a precipitate can be separated into elastic and plastic components. Using Eshelby's equivalent inclusion scheme (Mura, 1987), the increment in equivalent eigenstrains, inside a precipitate, can be obtained from the solution of the following set of linear equations,

$$
C_{i j k l}^{*}\left(d \varepsilon_{k l}^{o}+S_{k l m n} d \varepsilon_{m n}^{* *}-d \varepsilon_{k l}^{P r}\right)=C_{i j k l}\left(d \varepsilon_{k l}^{o}+S_{k l m n} d \varepsilon_{m n}^{* *}-d \varepsilon_{k l}^{* *}\right)
$$

Here, $d \varepsilon_{k l}^{o}$ is the increment in strains resulting from the applied stress. The increment in fictious eigenstrains $d \varepsilon_{i j}^{*}$ introduced in the equivalent inclusion method can be obtained as,

$$
d \varepsilon_{i j}^{*}=d \varepsilon_{i j}^{* *}-d \varepsilon_{i j}^{P r}
$$

The increment in elastic strains, inside a precipitate, can be expressed as,

$$
d \varepsilon_{i j}^{e l}=\left(S_{i j k l} d \varepsilon_{k l}^{* *}-d \varepsilon_{i j}^{P r}\right)
$$

Similar to the case of an elastic system, the stresses and strains inside an elastic-plastic precipitate are also uniform provided the matrix response is linear elastic. The total (elastic plus plastic) work done measured with respect to the loaded configuration, per unit volume of the precipitate, can be expressed as,

$$
W^{p r}=\frac{1}{2} \sigma_{i j} \varepsilon_{i j}^{e l}+\int_{o}^{\varepsilon_{i j}^{p}}\left(\sigma_{i j}+\sigma_{i j}^{o}\right) d \varepsilon_{i j}^{p}
$$

As described earlier in section $2, \sigma_{i j}^{o}$ is the remotely applied stress in a material that is free from any inclusion. $\sigma_{i j}$ is the sum of stress caused by the inhomogeneity and the internal stress arising from the prescribed eigenstrains. Since the increment in plastic strains $d \varepsilon_{i j}^{p}$ are derived from the total stresses $\left(\sigma_{i j}+\sigma_{i j}^{o}\right)$ via the constitutive relations, together they constitute a work-conjugate pair. Similarly, the elastic strains inside a precipitate $\varepsilon_{i j}^{e l}$ and the stress $\sigma_{i j}$ produce the elastic work.

The elastic strain energy of the matrix, per unit volume of precipitate, measured with respect to the loaded configuration can be obtained as (Eshelby, 1957),

$$
W^{M a t}=-\frac{1}{2} \sigma_{i j} S_{i j k l} \varepsilon_{i j}^{* *}+\frac{1}{2} \sigma_{i j}^{o} \varepsilon_{i j}^{*}
$$


The above set of equations are implemented numerically using an implicit predictor-corrector scheme. Full details of numerical implementation will be discussed elsewhere. Three different loading paths, as shown in fig.12, are analyzed. For load path-1, it is assumed that the phase transformation occurs first followed by the application of a gradually increasing external stress. This loading sequence is reversed for load path-2 whereas for load path-3 both external stress and the transformation strains are ramped up linearly. Load path-2 is more representative of the actual reactor service conditions. Hydrides are precipitated while cooling (during reactor shutdown) under a constant external stress.

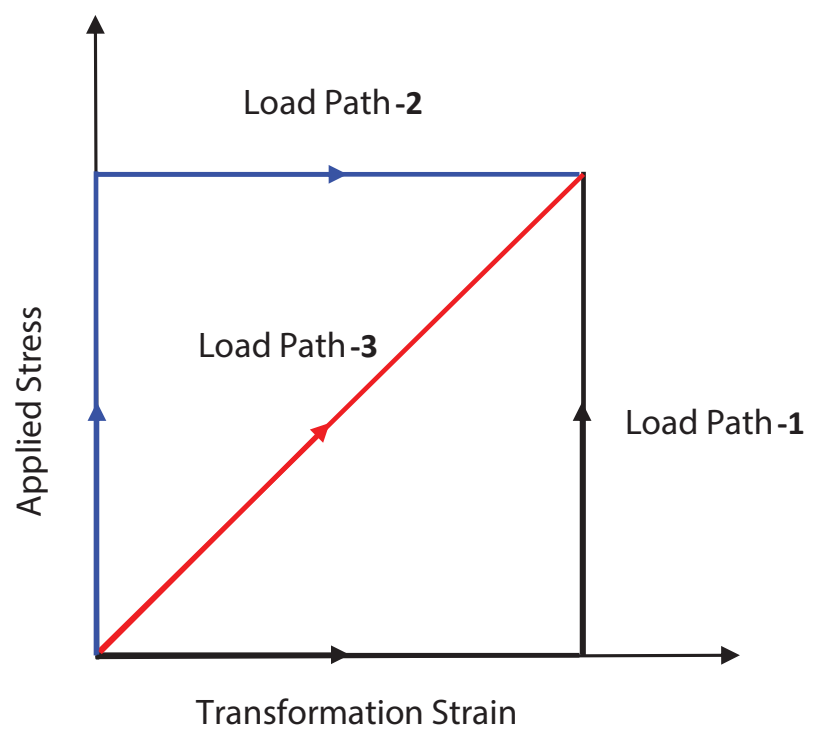

Figure 12: Schematic describing the loading paths considered in the analysis of an elastic-plastic hydride precipitate lying in an elastic zirconium matrix.

Numerical results presented in this sub-section are based on an assumption that applied stress is axi-symmetric, that is, $\sigma_{11}^{o}=\sigma_{22}^{o}$ and $\sigma_{33}^{o}=0$. An ellipsoidal shape, $c / a=0.1$, is assumed for a hydride precipitate. These assumptions are invoked so that simple axi-symmetric finite element analyses can be used to validate the numerical implementation. An interested reader may refer the work of Leitch and Puls (1992) etc. to gain further details on modeling of hydride precipitation using finite element analysis. The precipitate is modeled as elastic-perfectly plastic with a yield strength of $500 \mathrm{MPa}$. Both elastic as well as plastic response of the precipitate is assumed to be isotropic. A transversely isotropic elastic behavior is assumed for the $\alpha-\mathrm{Zr}$ matrix. Mismatch in elastic moduli of the matrix and precipitate is considered and numerical studies are performed for $f=0.5$. The major axis of the precipitate is assumed to lie in the basal plane $\left(\phi=0^{\circ}\right)$ of the matrix. The applied stress is assumed to be $1000 \mathrm{MPa}$. Transversely isotropic eigenstrains resulting from the $\delta$ hydride precipitation (Carpenter, 1973) are used in the numerical calculations. 


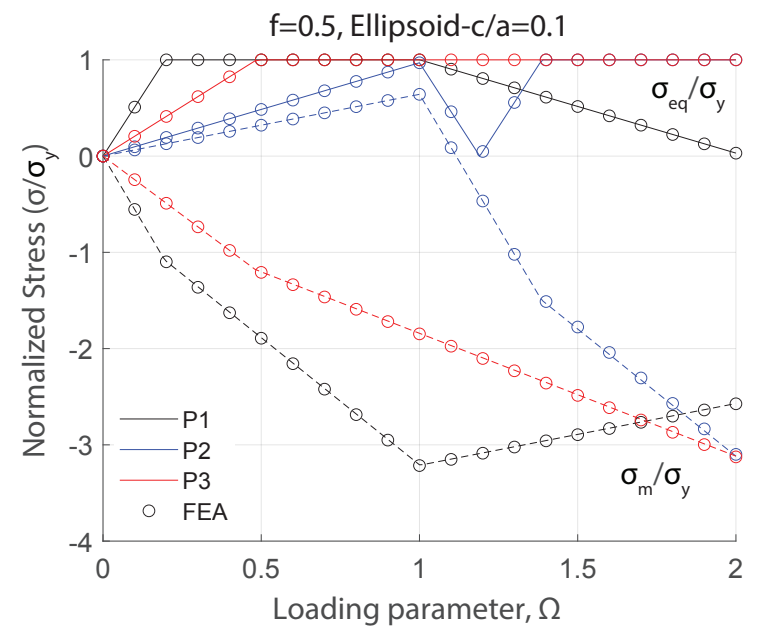

(a)

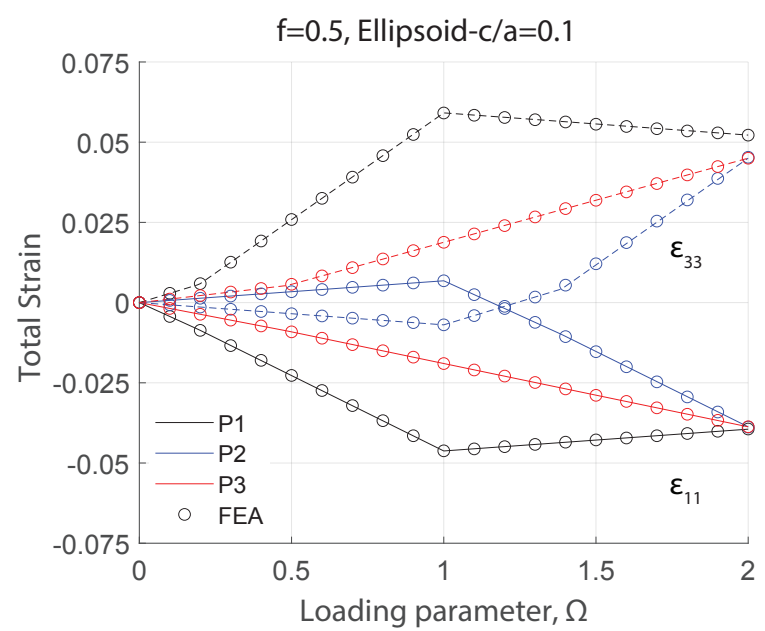

(b)

Figure 13: (a) Von Mises stress $\left(\sigma_{e q}\right)$ and mean stress $\left(\sigma_{m}\right)$ inside an elastic-plastic precipitate, obtained from the proposed numerical scheme, shown as solid and broken lines, respectively. The open circles denote the corresponding values obtained from an axi-symmetric finite element analysis. Three different loading paths denoted by $P_{1}, P_{2}$ and $P_{3}$ are considered. $\sigma_{y}$ is the yield strength of the precipitate. Similarly, results of total strain along the basal plane $\left(\varepsilon_{11}\right)$ and in the transverse direction $\left(\varepsilon_{33}\right)$, inside the precipitate, are shown in (b). Transversely isotropic eigenstrains resulting from the $\delta$ hydride precipitation Carpenter (1973) are used

Effect of loading path on the evolution of total stresses and total (elastic plus plastic) strains, inside a precipitate, is presented in fig.13(a) and (b), respectively. These are the combined variables resulting from the phase transformation and external stress. The Von-Mises stress $\left(\sigma_{e q}\right)$ and mean stress $\left(\sigma_{i i} / 3\right)$, obtained from the proposed numerical scheme, are shown as solid and broken lines, respectively. Corresponding results from finite element analysis are represented by the open circles. For load path-1, as the eigenstrains are ramped up, the initial response is linear elastic. Once the equivalent stress reaches the yield limit, plastic deformation occurs inside the precipitate. This transition from an elastic to plastic response is reflected as the change in slope of the mean stress. With further increase in eigenstrains, the mean stress keeps on increasing whereas the equivalent stress remains at $500 \mathrm{MPa}$. The loading parameter $\Omega=1$ marks the end of first load step; then the external stress is gradually increased from 0 to $1000 \mathrm{MPa}$. The application of an axi-symmetric tensile stress reduces the compressive stress generated in the precipitate due to the phase transformation. As a result, the mean stress decreases as the external stress is increased. The equivalent stress also shows a corresponding reduction until the end of the second load step. For load path-2, the precipitate is in tension and remains in an elastic state as the magnitude of applied stress is ramped up. The first load step ends at $\Omega=1$; then the eigenstrains are prescribed. The compressive stresses generated due to eigenstrains dominate over the external tensile stress and the hydrostatic stress changes from tension to compression. The equivalent stress also decreases and then starts increasing until the precipitate yields in compression. For load path-3, similar to the case of path-1, the precipitate remains in compression from the beginning, although the magnitude of the compressive stresses is lower. It is observed that load path-2 and 3 lead to nearly the same values of equivalent and 
mean stress in the final state, whereas these stresses are substantially lower for load path-1. Plots of total strains inside the precipitate are shown in fig.13(b).

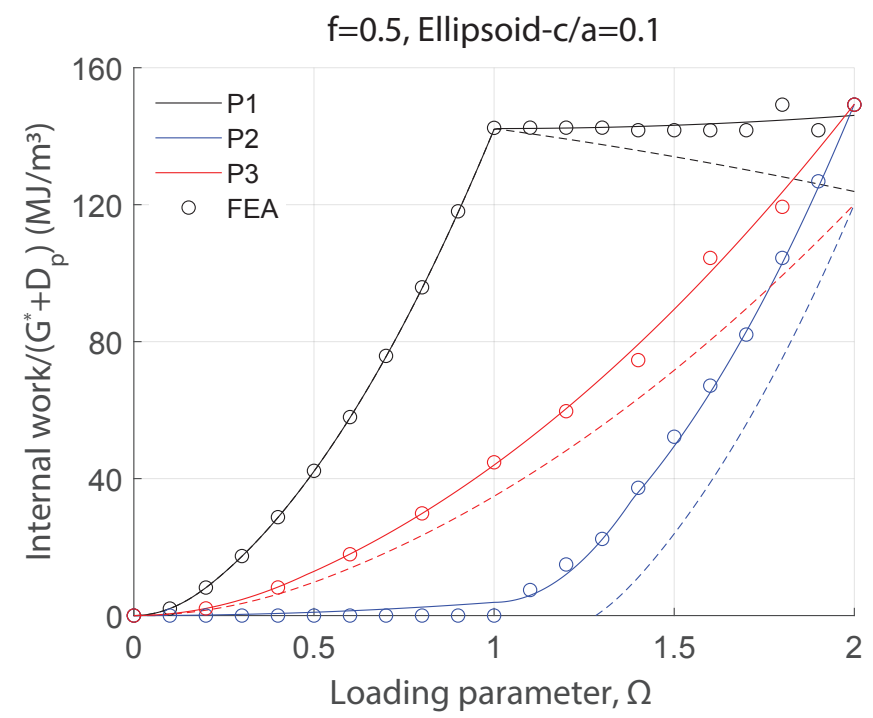

Figure 14: Internal work, and the sum of Gibbs free energy and plastic dissipation $G^{*}+D_{p}$ per unit volume of a hydride precipitate, obtained from the proposed numerical scheme, shown as solid and broken lines, respectively. The open circles denote the results of internal work obtained from axi-symmetric finite element analysis. Three different loading paths denoted by $P_{1}, P_{2}$ and $P_{3}$ are considered. Both internal work and $G^{*}+D_{p}$ are measured with respect to the initial loaded and untransformed state. Transversely isotropic eigenstrains resulting from the $\delta$ hydride precipitation Carpenter (1973) are used

We now analyze the effect of loading path on the energetics and dissipation associated with hydride precipitation, see fig.14. For an elastic system, the total energy is the sum of the elastic strain energy of the body and the potential energy of the external stress and corresponds to the Gibbs free energy of the body (Mura, 1987). For an elastic-plastic precipitate, a part of the mechanical energy is stored in the form of elastic strain energy and the rest goes in plastic dissipation, see eq.(32). The total energy of the precipitate and matrix will be referred to as the internal work. Note that only a part of this internal work is recoverable. The potential energy of applied stress is determined using the same scheme as used for the elastic system. Since the plastic strain is treated as a transformation strain, the potential energy of the external stress and the recoverable part of the internal work together constitute the Gibbs free energy. Since the precipitate is undergoing both elastic and plastic deformations, the Gibbs energy is accompanied by the plastic dissipation. A phase transformation is feasible provided the chemical (phase) energy compensate for any increase in the Gibbs free energy $G^{*}$ and the energy dissipated by plastic deformation $D_{p}$. The configuration that minimizes $G^{*}+D_{p}$ will be the most preferred one. The sum of internal work and the potential energy of the external stress provide us this quantity.

For an applied axi-symmetric stress of $1000 \mathrm{MPa}$, the internal work obtained from the three 
different loading paths is almost the same. Apparently, the result may look a bit surprising since the stresses inside the precipitate are observed to be substantially lower for load path-1, see fig.13(a). The total work done on the precipitate, in fact, is lower for this case. It is, however, the in-plane stresses which are of smaller magnitude as compared to the other two loading paths. The transverse component $\left(\sigma_{33}\right)$ is actually higher for load path-1. Since the matrix experiences largest strain in the transverse direction, the relatively higher value of $\sigma_{33}$ for load path-1 and the energy of inhomogeneity (second term in eq.(33)) results in higher strain energy of the matrix. As a result, the internal work is not much different for the three load paths, see fig.14. The combined sum of the Gibbs free energy and the plastic dissipation, that is, $G^{*}+D_{p}$ is shown as broken lines in fig.14. In particular, load path-2 leads to slightly lower values of $G^{*}+D_{p}$. Thus, for the practical range of applied stress to which structural components may be subjected, a significant effect of loading path is not expected. Slight differences in the numerical values of internal work obtained from our proposed scheme and those of finite element analyses can be observed. This is due to the numerical errors involved in separating out the work done on the hydride precipitate from that of the loaded configuration, during post processing of finite element results.

\section{Morphology and orientation of an elastic-plastic hy- dride precipitate}

Our studies on an elastic-plastic precipitate lying in an elastic matrix have revealed that, for the practical range of applied stress, the effect of loading path on the energetics and dissipation associated with hydride precipitation is not significant. We, therefore, prefer to choose load path-2 as it is more representative of the actual service conditions under which hydride precipitation occurs in structural components. We begin with analyzing the effect of elastic mismatch factor $f$ on the morphology and orientation of an elastic-plastic hydride precipitate. The matrix behavior is assumed to be elastic and transversely isotropic. The plot of normalized internal work $W^{*} / E \delta^{2}$ for a hydride precipitate is shown in fig. 15 .

For a spherical precipitate, the internal work is not much different from the elastic case, see fig.6. Since the state-of-stress inside a sphere is nearly hydrostatic, the plastic deformation is limited. Had the transformation strains and the matrix behavior been isotropic elastic, the results of an elastic-plastic precipitate would have been identical to that of the elastic case. With decreasing aspect ratio, shear stresses develop inside the precipitate leading to plastic relaxation. Results for a needle shaped precipitate are close to that of a sphere. The effect of precipitate orientation, for a given shape, on the internal work is also analyzed. For both an overmatched $(f>1)$ and an undermatched $(f<1)$ case, a disc shaped precipitate lying in the basal plane, $\phi=0^{\circ}$, minimizes the internal work. For a needle shaped precipitate, the orientations corresponding to the minimum internal work are $\phi=45^{\circ}$ and $39^{\circ}$ for $f=0.5$ and 3 , respectively. 
Transversely isotropic elastic matrix - Elastic-Plastic precipitate

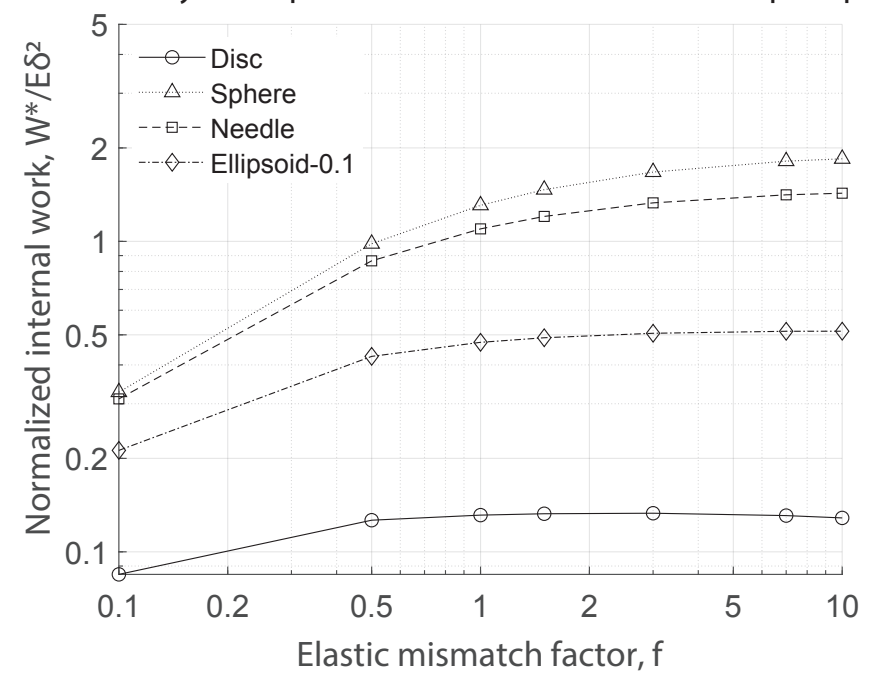

Figure 15: Effect of elastic mismatch on the internal work $W^{*}$ for an elastic-plastic hydride precipitate lying in elastic zirconium matrix. Four different shapes of precipitate namely disc, sphere, needle and an ellipsoid with aspect ratio $c / a=0.1$ are analyzed. Eigenstrains $\epsilon_{11}=\epsilon_{22}=0.0458$ and $\epsilon_{33}=0.072$ are used in the numerical calculations

We now analyze the effect of a uniaxial applied stress on the morphology and orientation of an elastic-plastic hydride precipitate. As before, the applied stress is assumed to act either in the transverse direction $\left(\sigma_{33}^{o}\right)$ or along the basal plane $\left(\sigma_{11}^{o}\right)$. Numerical results are presented for a disc and needle shaped precipitate. The sum of Gibbs free energy and plastic dissipation in a normalized form $\left(G^{*}+D_{p}\right) / E \delta^{2}$ for an undermatched precipitate $(f=0.5)$ is shown in fig.16(a). In contrast to the elastic case, the quantity $G^{*}+D_{p}$ for a thin disc is substantially lower than that of a needle. This is essentially due to the high hydrostatic stress in the latter case. The effect of loading direction on $G^{*}+D_{p}$ is insignificant for both disc and needle shaped precipitates. Such a behavior is expected as the plastic flow inside the precipitate is assumed to be isotropic. Plots of $G^{*}+D_{p}$ for a disc and needle shaped precipitate for an overmatched case $(f=3)$ look very similar to those of an undermatched case $(f=0.5)$ and are, therefore, omitted. The reason behind this similarity is, however, worth discussing. When an elastic system is subjected to a high magnitude of applied stress, it is the energy of an inhomogeneity that governs the total potential energy, $G^{*}$. For an isotropic elastic matrix, Pineau (1976) has demonstrated that the energy of an inhomogeneity varies quadratically with applied stress and it is negative for an undermatched case and positive for an overmatched case. This is precisely the reason for potential energy plots to appear as inverted and upward parabolas for the two cases, see fig.4. For an elastic-plastic precipitate, it is, however, the interaction energy of an external stress that dominates $G^{*}+D_{p}$. For both undermatched and overmatched cases, the interaction energy is observed to be negative and, therefore, the plots of $G^{*}+D_{p}$ for the two cases look similar, although the numerical values are different. The orientations corresponding to the minimum $G^{*}+D_{p}$ for an undermatched disc shaped precipitate are presented in fig.16(b). At low magnitude of applied stress, the results are very similar to that of the elastic case, see fig.8(b). As the applied stress increases, the effect of transformation strains quickly decay and 
the orientations minimizing $G^{*}+D_{p}$ become independent of the applied stress. The case of an overmatched $(f=3)$ disc shaped precipitate also exhibits a very similar behavior. For the case of an external stress acting along the basal plane, a stress of $100 \mathrm{MPa}$, that is, $\sigma_{11}^{o} / \sigma_{y}=0.2$, causes a complete reorientation of an elastic-plastic hydride precipitate.

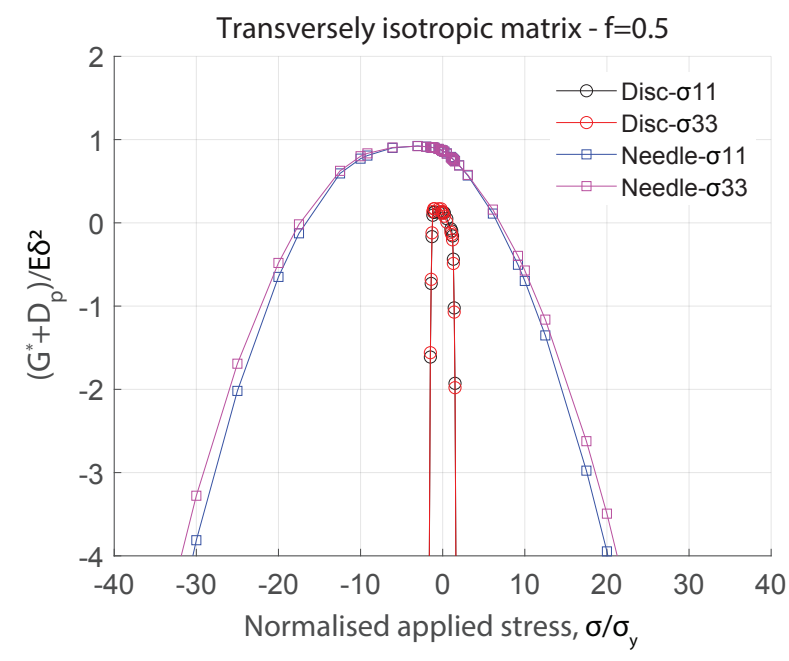

(a)

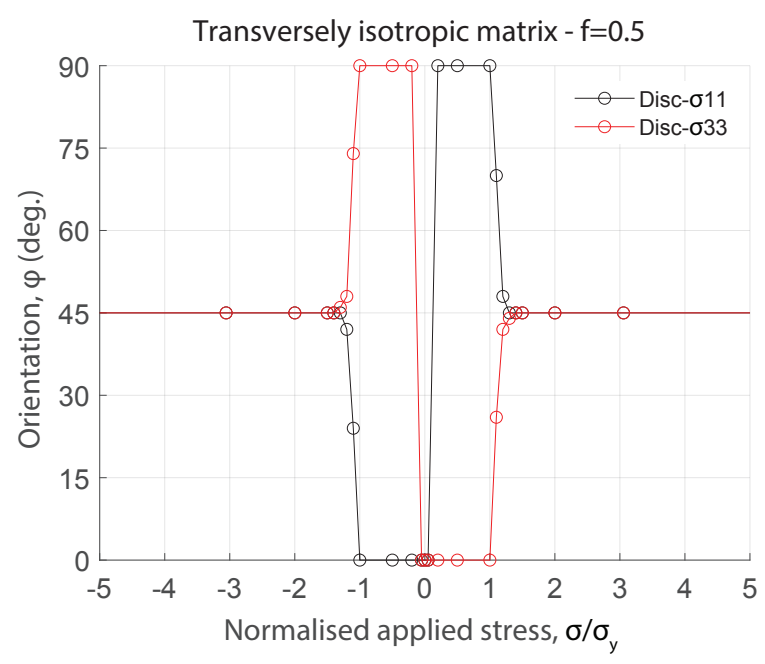

(b)

Figure 16: (a) Sum of Gibbs free energy and plastic dissipation $G^{*}+D_{p}$ for an elastic-plastic disc and needle shaped precipitate, as a function of applied uniaxial stress, for an undermatched case $(f=0.5)$, (b) Orientation of a disc shaped precipitate that minimizes $G^{*}+D_{p} . \sigma_{y}$ is the yield strength of a hydride precipitate, taken as $500 \mathrm{MPa}$. Eigenstrains $\epsilon_{11}=\epsilon_{22}=0.0458$ and $\epsilon_{33}=0.072$ are used in the numerical calculations

The orientations minimizing $G^{*}+D_{p}$ for a needle shaped precipitate are shown in fig.17. As observed for the case of a thin disc, the orientation plot for an overmatched case $(f=3)$ is similar to that of an undermatched precipitate $(f=0.5)$, the numerical values are of course different. 


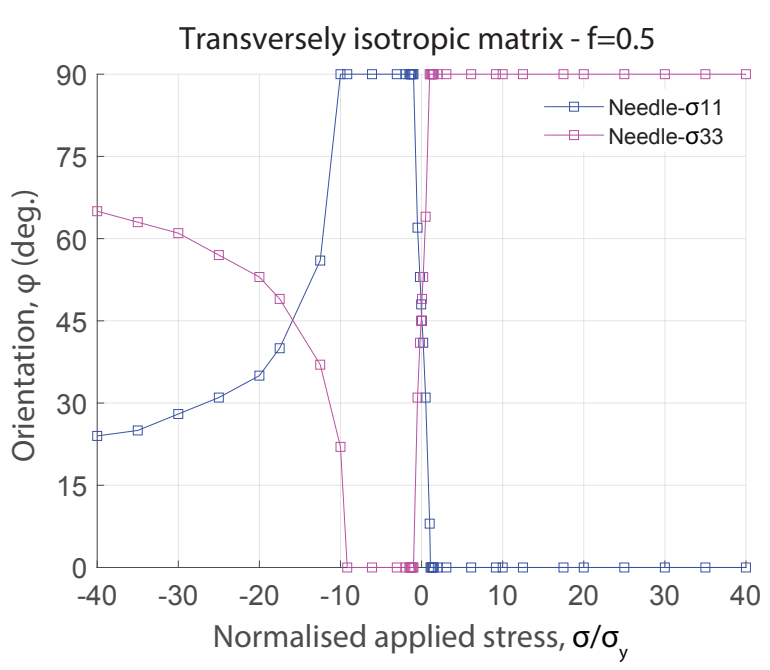

(a)

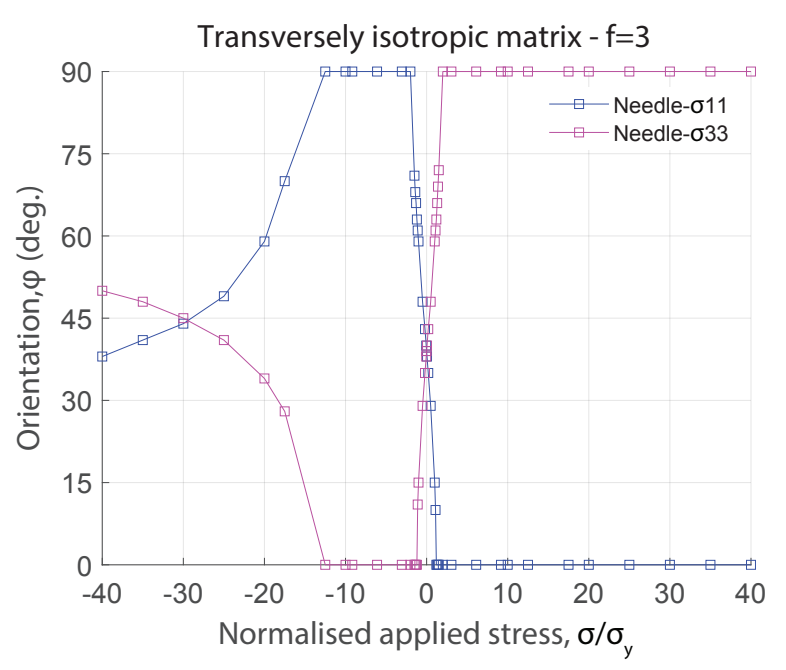

(b)

Figure 17: Orientation of an elastic-plastic needle shaped precipitate corresponding to minimum $G^{*}+D_{p}$ for an elastic mismatch factor (a) $f=0.5$ and (b) $\mathrm{f}=3$, respectively. Stress $\sigma_{y}$ is the yield strength of a hydride precipitate, taken as $500 \mathrm{MPa}$. Eigenstrains $\epsilon_{11}=\epsilon_{22}=0.0458$ and $\epsilon_{33}=0.072$ are used

Several experimental investigations on hydride reorientation in zirconium based alloys have been reported in the literature. Test data of unirradiated, cold worked and stress-relieved Zircaloy-4 suggest that the threshold stress under uniaxial tension is in the range of 140-200 $\mathrm{MPa}$ (Cinbiz et al., 2016) whereas it is in the range of 190-230 MPa for Zr-2.5wt\% Nb alloy (Leger and Donner, 1985). The latter authors attributed the increase in threshold tensile stress to the relatively higher yield strength of $\mathrm{Zr}-2.5 \mathrm{wt} \% \mathrm{Nb}$.

At this point, it is worth mentioning some of the issues associated with reorientation of hydride precipitates. Most of the experimental studies on hydride reorientation have focused on macroscale hydrides that appear as platelet-shaped entities under an optical microscope. Recent studies, however, have revealed that the macroscale reoriented hydrides comprises a stacking of several microscopic platelets that maintain their orientation parallel to the basal plane (Motta et al., 2019; Han et al., 2019). Different hypothesis have been proposed in the literature to explain the macroscale hydride reorientation. Une et al. (2004) suggested that the external tensile stress causes a change in the preferred precipitation sites of both intra and inter-granular hydrides, the crystallographic relationship between the hydride and matrix remains unchanged. The hydrides tend to preferentially precipitate in the basal planes and grain boundaries facing the tensile stress. Eventually, they interlink with each other forming radial strings of the hydride as observed by optical microscopy. Based on a phase field study, Han et al. (2019) proposed that the stress-induced heterogeneous nucleation and growth, near a pre-existing hydride, results in the formation of a unique stacking structure that appears as a reoriented macro-hydride. The analysis reported in the present study discusses the reorientation of a single hydride platelet lying in an $\alpha$-zirconium grain. Although experimental studies suggest that within a radially oriented macroscale hydride the individual nanoscale precipitates lie in the basal plane of zirconium matrix, phase field studies, however, lead to a complete reorientation of a single hydride precipitate (see Motta et al., 2019). Since the constitutive response of the 
matrix and precipitate was modeled as linear elastic in these studies, the threshold stresses were quite high as compared to the experimental values. Incorporation of precipitate plasticity in the numerical calculations leads to hydride reorientation at realistic magnitudes of applied stress. Our elastic calculations presented in section 4.4, however, have already indicated that the anisotropy in the interfacial energy plays a significant role in controlling the orientation of a hydride precipitate.

We conclude this section by analyzing the effect of anisotropic interfacial energy on the orientation of an elastic-plastic disc shaped hydride precipitate subjected to an external stress, see fig.18. For a wide range of applied stress, similar to the case of an elastic precipitate, the strong anisotropy in the interfacial energy causes a hydride precipitate to remain in the basal plane. Since, the internal work for a plastic precipitate is significantly smaller than the elastic strain energy, the effect of interfacial energy on the orientation of a plastic precipitate diminishes at a much larger size of hydride as compared to the elastic case, see fig.11.

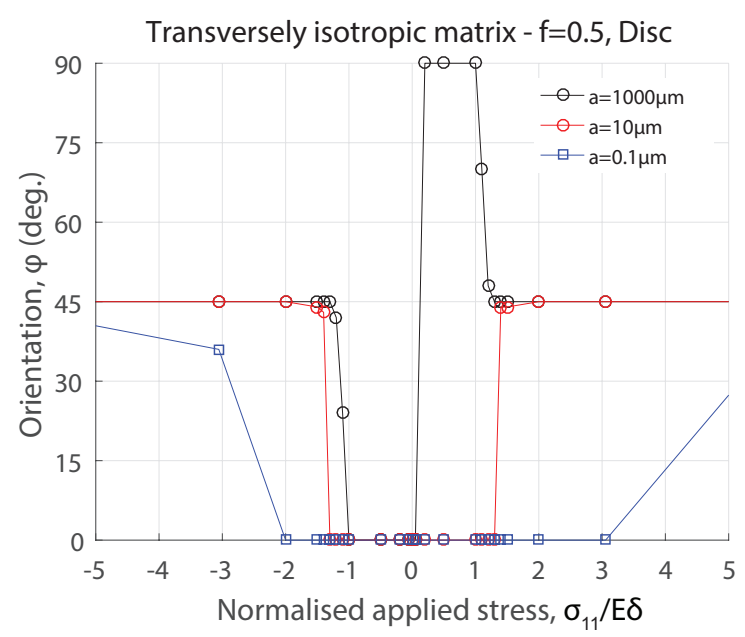

Figure 18: Effect of anisotropic interfacial energy on the orientation of a disc shaped hydride precipitate corresponding to minimum Gibbs free energy $G^{*}$. An elastic-plastic precipitate lying in elastic zirconium matrix is analyzed. Parameter $a$ is the length of semi-major axis of the precipitate. Eigenstrain $\epsilon_{11}=\epsilon_{22}=0.0458$ and $\epsilon_{33}=0.072$ are used in the numerical calculations

\section{Hydride reorientation in an elastic-plastic zirconium matrix}

In this section, an assessment of matrix plasticity on the mechanics of hydride reorientation under an external stress is carried out. Plane-strain finite element analyses are performed on an elastic-plastic hydride precipitate lying in a plastically deforming zirconium matrix. Both precipitate and matrix are modeled as elastic-perfectly plastic (non-hardening). The elastic properties of $\delta$-hydride and the $\alpha$-zirconium matrix and the anisotropic eigenstrains are the same as described in section 4. Numerical results are presented for the case $f=1$. Based on the recent work of Sharma et. al. (2018), the yield strength of $\delta$-hydride and $\alpha$-zirconium are 
taken as $325 \mathrm{MPa}$ and $450 \mathrm{MPa}$, respectively. Isotropic $J_{2}$ flow theory is used to describe the plastic response of hydride and zirconium matrix. An external stress is applied parallel to the basal plane. Two limiting orientations, that is, a hydride lying parallel to the external stress $\phi=0^{\circ}$ and perpendicular to the external stress $\phi=90^{\circ}$ are analyzed. The hydride is modeled as a flat ellipsoid with an aspect ratio $c / a=0.1$. The size of matrix modeled in our analysis is large in comparison to the hydride size to minimize the boundary effects. Sufficiently refined finite element meshes are used to discretize the region near the matrix-hydride interface. In the absence of external stress, the total internal work per unit volume of precipitate for the case when the hydride is lying in the basal plane is around $93 \mathrm{MJ} / \mathrm{m}^{3}$, which is in good agreement with the numerical results of Sharma et al. (2018). Due to anisotropy in elastic properties of the zirconium matrix and anisotropic eigenstrains, the internal work increases with orientation angle $\phi$ and attains a value of $98.9 \mathrm{MJ} / \mathrm{m}^{3}$ at $\phi=90^{\circ}$. The application of an external stress acting along the basal plane causes a small increase in the values of internal work, see table 1.

Table 1: Internal work and the sum of Gibbs free energy and plastic dissipation $\left(G^{*}+D_{p}\right)$ for a hydride precipitate lying in $\alpha$-zirconium matrix. Both hydride and matrix are modeled as elastic-perfectly plastic. An external stress $\sigma_{11}^{o}$ is applied along the basal plane.

\begin{tabular}{cccc}
\hline $\begin{array}{c}\text { Applied stress } \\
(\mathrm{MPa})\end{array}$ & $\begin{array}{c}\text { Orientation angle } \\
\phi\end{array}$ & $\begin{array}{c}\text { Internal work } \\
\left(\mathrm{MJ} / \mathrm{m}^{3}\right)\end{array}$ & $\begin{array}{c}G^{*}+D_{p} \\
\left(\mathrm{MJ} / \mathrm{m}^{3}\right)\end{array}$ \\
\hline 0 & $0^{\circ}$ & 93 & - \\
0 & $90^{\circ}$ & 98.9 & - \\
\hline 200 & $0^{\circ}$ & 98.06 & 84.35 \\
200 & $90^{\circ}$ & 101.4 & 46.37 \\
\hline
\end{tabular}

A plot of Von Mises and hydrostatic stress in and near the hydride region for the two different orientations is shown in fig.19. Due to plastic deformation in the matrix, the stresses inside the hydride precipitate are not uniform. A higher value of hydrostatic stress inside the precipitate and at the tip of the hydride is observed for $\phi=90^{\circ}$. Our numerical calculations reveal that the external work done by the applied stress plays an important role in the mechanics of hydride reorientation. For the case when the precipitate is normal to the applied stress $\left(\phi=90^{\circ}\right)$, the work done by the applied stress is almost four times higher than that obtained for the case of $\phi=0^{\circ}$. As a result, the sum of Gibbs free energy and plastic dissipation $G^{*}+D_{p}$ is significantly lower for $\phi=90^{\circ}$, thus, indicating that a hydride precipitate will completely reorient at an external stress of $200 \mathrm{MPa}$. The numerical results (presented in section 6) obtained from our simplified scheme are, therefore, in agreement with the more detailed results based on a treatment of precipitate and matrix plasticity. As discussed earlier, the anisotropy in interfacial energy, however, will not allow the reorientation of a precipitate. 


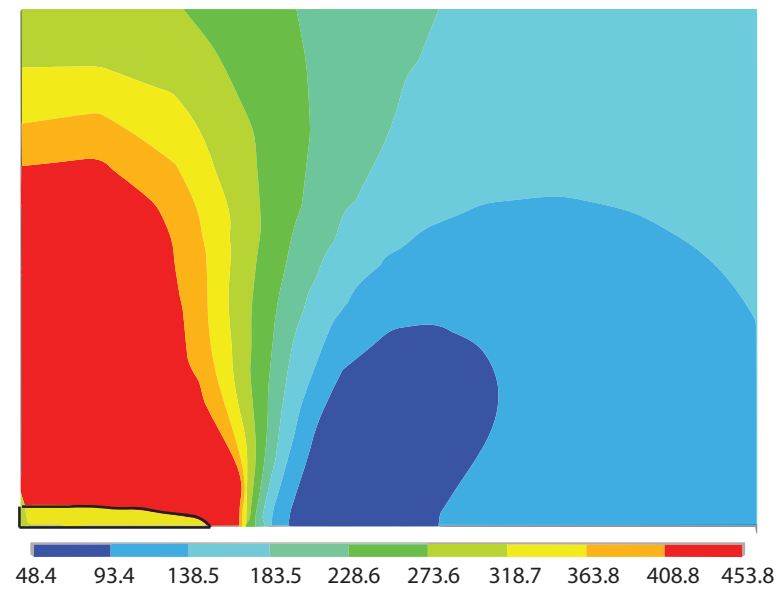

(a)

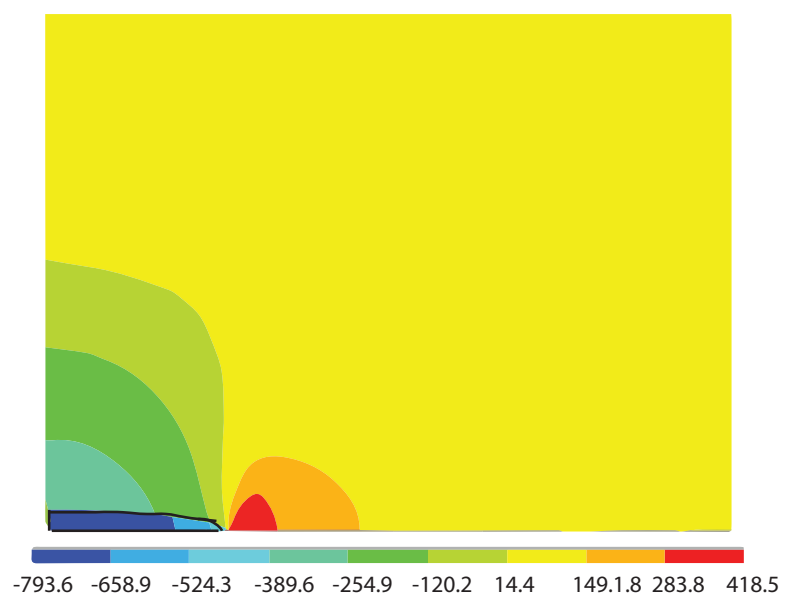

(c)

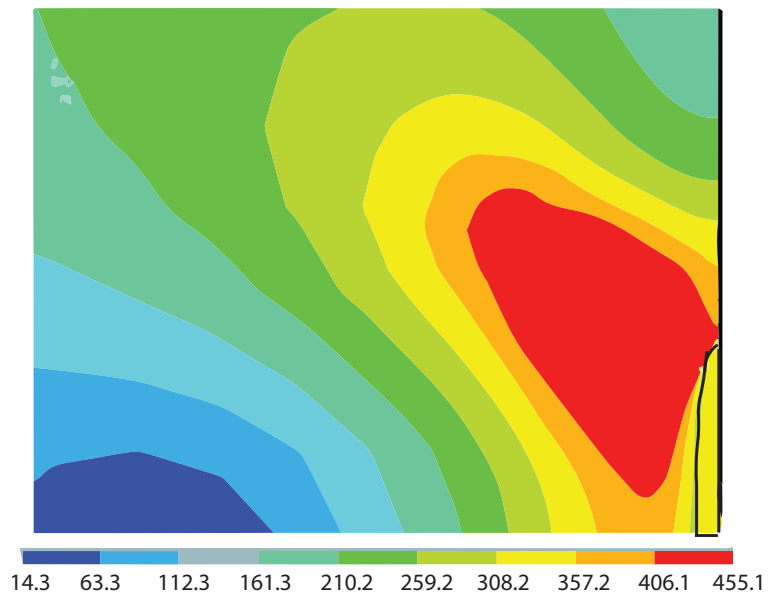

(b)

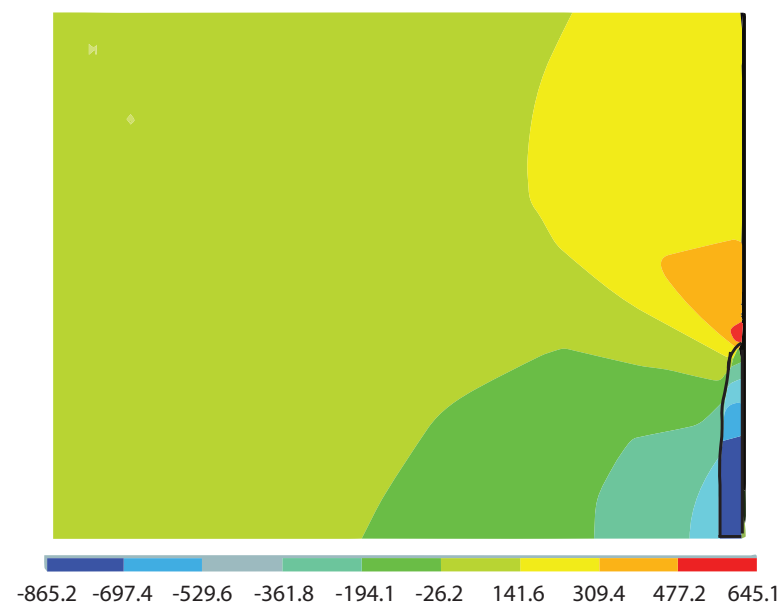

(d)

Figure 19: Contour plots of Von Mises stress in and near the hydride region for the case when a precipitate is lying parallel $\left(\phi=0^{\circ}\right)$ and perpendicular to the external stress $\left(\phi=90^{\circ}\right)$ are shown in (a) and (b), respectively. An external stress of $200 \mathrm{MPa}$ is acting along the basal plane. Corresponding plots for hydrostatic stress are shown in (c) and (d), respectivly. Eigenstrains $\epsilon_{11}=\epsilon_{22}=0.0458$ and $\epsilon_{33}=0.072$ are used.

\section{Discussion}

Hydride induced embrittlement in zirconium alloys has a detrimental effect on the structural integrity of components used inside the reactor core. Although a clear understanding of the mechanism of hydride precipitation in zirconium alloys does exist, the ability to predict their formation is poor. The hydride phase so precipitated has a tendency to reorientate under an external stress (see Bair et al., 2015; Motta et al., 2019), and this phenomenon is considered to be largely responsible for the embrittlement of zirconium alloys (Puls, 2012). Detailed studies addressing the energetics and dissipation associated with hydride precipitation are lacking. The present manuscript attempts to bring some clarity to these aspects.

In this study, a numerical scheme for analyzing an inhomogeneous inclusion in an anisotropic elastic medium is developed. The proposed scheme is based on Eshelby's solution and the 
effect of elastic mismatch, anisotropic eigenstrains resulting from phase transformation and an external stress are accounted for. The problem of an inhomogeneous inclusion in an isotropic elastic matrix is first considered. Assuming the eigenstrains to be purely dilatational, closedform expressions describing the effect of elastic mismatch between the matrix and precipitate and the applied external stress on the orientation of disc and needle shaped precipitates are proposed. Our studies reveal that simple orientations, such as a disc perpendicular to an applied stress or a needle aligned with the stress axis, are not always the configurations of minimum potential energy. For a fixed value of dilatational eigenstrain $(\delta)$, the stress required to cause reorientation of disc and needle shaped precipitates depends on the elastic constants of the matrix and the elastic mismatch factor $f$. At high values of applied stress, the effect of transformation strain decays and the orientation of an elastic inhomogeneity is independent of the nature (tensile/compressive) and magnitude of applied stress.

A detailed elastic analyses of a hydride precipitate in a transversely isotropic zirconium matrix is then carried out. The role of elastic mismatch and applied stress in controlling the morphology and orientation of a hydride precipitate is analyzed. The effect of applied stress direction with respect to matrix crystallographic planes is accounted for. Unlike the case of an isotropic matrix, a complete reversal of orientation when the stress transitions from tension to compression, or vice-versa, is not observed. There exists a threshold stress, in some cases, that causes a sudden change in the orientation of a hydride precipitate. This is essentially a consequence of anisotropy in the elastic properties of the zirconium matrix and the anisotropic transformation strains resulting from hydride precipitation. At higher stress magnitudes, a second reorientation phenomenon is observed which is a consequence of the inhomogeneity effect. The influence of stress biaxiality on the orientation of a hydride precipitate is also analyzed. The application of a minor stress in the orthogonal direction results in an increase in the threshold tensile stress for an undermatched disc shaped precipitate. Although experimental studies of Cinbiz et al. (2016) have shown that the stress biaxiality leads to a significant reduction in the threshold stress, the authors could not provide a satisfactory explanation of this. A more complete treatment, accounting for plastic deformation of the precipitate and the matrix, under a biaxial stress may reveal some insights.

To overcome some of the restrictions arising from pure elastic analysis, we have proposed a numerical scheme accounting for plastic deformation in the precipitate. The matrix behavior is still modeled as anisotropic elastic. The role of elastic mismatch in controlling the morphology and orientation of an elastic-plastic precipitate is limited. Unlike the elastic case, the internal work for a disc shaped precipitate is substantially lower than that of a spherical or needle shaped precipitate. This is essentially due to the large shear stress that develops in the former case, thus, leading to plastic relaxation in the hydride. The numerical results obtained from our proposed simplified scheme are found to be in agreement with the more detailed finite element results that accounts for precipitate and matrix plasticity. For a wide range of elastic mismatch $(0.5<\mathrm{f} \leq 3)$, an applied stress of $\approx 100 \mathrm{MPa}$, acting along the basal plane, causes a complete reorientation of an elastic-plastic hydride precipitate. The above results are valid when the precipitates are large, so that the mechanical contributions to the Gibbs free energy are much larger than that from the interface. Incorporation of interfacial energy in the numerical calculations, however, reveals that for small hydrides the anisotropy in surface energy controls the orientation of a precipitate, thus causing it to be parallel to the basal plane. Thus, nucleated precipitates will always lie parallel to the basal plane, with mechanical work and interfacial energy considerations favouring the formation of disc shaped precipitates. As the precipitates 
grow the above analysis suggests that they can reorientate in response to an applied stress once they reach a critical size. In practice, individual precipitates are observed to always lie in the basal plane. The reason for this is that there needs to be a kinetic pathway from a small precipitate lying in the basal plane into another orientation. This requires there to be a positive driving force for the reorientation along the entire pathway, i.e. a smooth continuous decrease in free energy. In practice, the variation of interfacial energies with crystallographic orientation has a cusped profile centred on low energy configurations, (see Taylor and Cahn, 1986; Gill and Cocks, 2006). This gives rise to an infinite gradient of the free energy with respect to orientation of the precipitate and, therefore, an infinite negative driving force for reorientation, independent of the size of the precipitate. A precipitate, therefore, gets trapped in orientations determined by this cusped interfacial energy profile. Nonetheless, the system would always want to minimise its free energy. This is achieved in practice by the way in which the individual precipitates arrange within the body. Clusters of particles organise themselves into groups that can rearrange in response to the applied stress. The calculations presented here provide the essential building blocks for studying the reorientation of these clusters.

The eigenstrains employed in our numerical study were computed by Carpenter (1973) using the lattice parameters of $\alpha$-zirconium and $\delta$-hydride at room temperature. The temperature dependent eigenstrains, (see Sharma et al., 2018) may be easily incorporated in our numerical scheme to analyze the effect of precipitation temperature on the morphology and orientation of zirconium hydride.

We finally touch upon the applications of our proposed numerical schemes (Section 2 and 5) in other relevant fields. The problem of microstructural evolution is of current interest to the material science community. In solid state phase transformations, the elastic energy of the microstructure constitutes a substantial fraction of the total free energy. Of course, one approach for solving the elasticity equations is to adopt a direct numerical scheme like the finite element method used in conjunction with the phase field method. Such direct simulations are, however, expensive and analytical schemes like those developed by Eshelby (1957) are often employed to resolve the elastic fields within and around the microstructure. The computational scheme (Section 2) developed in this work appears to be suitable for analyzing phase transformation problems as it fully accounts for the matrix anisotropy, inhomogeneity of the precipitate and is valid for a general ellipsoidal shape. Moreover, an assessment of the effect of precipitate plasticity (Section 5) can also be carried out. Another potential application of our proposed schemes is in Geological Sciences. A number of investigations have been made in the past where Eshelby solutions were used for analysis of faulting and fracture patterns, and simulation of compaction bands. Since Eshelby solutions for an arbitrary ellipsoidal inhomogeneity requires extensive calculations, an assumption of isotropic matrix is often invoked. Our proposed scheme, that eliminates the need for such an assumption, is expected to provide more accurate solutions to some of these problems. Perhaps another interesting application would be the utilization of our computational schemes in conjunction with an averaging technique like Mori-Tanaka to evaluate the instantaneous effective properties of a composite.

\section{Conclusions}

Studies carried out in this work suggest that:

(a) If surface energy effects are insignificant, the mismatch in elastic moduli of the matrix 
and inclusion plays a central role in controlling the morphology and orientation of a precipitate. Simple orientations, such as a disc perpendicular to an applied stress or a needle aligned with the stress axis, are not always the configurations of minimum potential energy.

(b) For a range of elastic mismatch between a hydride precipitate and the zirconium matrix, $f \leq 1.5$, either a disc or a flat ellipsoid (aspect ratio $c / a \approx 0.1$ ) lying in the basal plane minimizes the elastic strain energy. These configurations are in agreement with the morphology of hydride precipitates observed in experiments (Motta et al., 2019).

(c) In the absence of surface energy, the minimum Gibbs free energy of a disc shaped hydride precipitate, under an external stress, is observed to occur at different crystallographic planes of the zirconium matrix. When the external stress acts along the basal plane, there exists a threshold tensile stress that causes a complete reorientation of an undermatched hydride precipitate. For a wide range of elastic mismatch $(0.5<\mathrm{f} \leq 3)$, an applied stress of $\approx 100 \mathrm{MPa}$, acting along the basal plane, causes a complete reorientation of a disc shaped elastic-plastic hydride precipitate.

(d) For a small hydride, the strong anisotropy in interfacial energy controls the orientation of a disc shaped precipitate causing it to remain parallel to the basal plane. As a precipitate grows, it can be trapped in this orientation due to the cusp shaped profile of the way in which the interfacial energy changes with orientation.

\section{Acknowledgement}

The work reported in this study was initiated during a stay of the first author (IAK) in the Department of Engineering Science, University of Oxford, UK as a Marie Sklodowska-Curie fellow under the European Union's Horizon 2020 Research and Innovative program. The funding received under the grant agreement No. 701897 is gratefully acknowledged. The authors are grateful to the anonymous reviewers for bringing some relevant phase field studies and experimental investigations to our knowledge. Constant encouragement and invaluable support provided by Mr. Vivek Bhasin, Director, Nuclear Fuels Group, BARC is acknowledged.

\section{Appendix A.1 Potential energy of an inhomogeneous inclusion in an isotropic elastic matrix}

We present here some details of an analytical scheme used to evaluate the total potential energy of an inhomogeneous inclusion in an isotropic elastic matrix. The eigenstrains are assumed to be purely dilatational, that is, $\varepsilon_{11}^{p}=\varepsilon_{22}^{p}=\varepsilon_{33}^{p}=\delta$. The Eshelby equivalent inclusion method, eq.(9), can be expressed in the following form,

$$
\left[\left(C_{i j k l}^{*}-C_{i j k l}\right) S_{k l m n} \varepsilon_{m n}^{* *}+C_{i j k l} \varepsilon_{k l}^{* *}\right]=\left[\left(C_{i j k l}-C_{i j k l}^{* *}\right) \varepsilon_{k l}^{o}+C_{i j k l}^{*} \varepsilon_{k l}^{p}\right]
$$

For numerical implementation, it will be easier to express the tensorial quantities in vector form using the standard Voigt notation. The above set of linear equations to determine the equivalent eigenstrains can then be expressed in the matrix form,

$$
[A]\{X\}=\{B\}
$$


Here $[A]$ is a $6 \times 6$ square matrix that is a function of the elastic constants of the matrix $\left(C_{i j k l}\right)$ and the precipitate $\left(C_{i j k l}^{*}\right)$, and the Eshelby tensor $\left(S_{i j k l}\right) .\{X\}$ is a $6 \mathrm{x} 1$ vector that describes the equivalent eigenstrains. $\{B\}$ is a $6 \times 1$ unknown vector that is a function of the elastic constants of the matrix and the precipitate, the prescribed eigenstrains and the strains arising from an externally applied stress field. We consider here the case of a uniaxial applied stress $\left(\sigma_{33}^{o}\right)$ and the orientation of the precipitate, relative to the stress axis, is defined by the Euler angle $\phi$ see fig.1. For an isotropic elastic system (precipitate and matrix) with dilatational eigenstrains, only the first term on the right hand side of eq.(A-1), denoted as $b_{i j}$, is a function of precipitate orientation. Using the standard tensor transformation rules, the vector $\{B\}$ in the local co-ordinate system of the precipitate, therefore can be expressed as,

$$
\{B\}_{\phi}=\left[\begin{array}{ccc}
c & 0 & -s \\
0 & 1 & 0 \\
s & 0 & c
\end{array}\right]\left[\begin{array}{lll}
b_{11} & b_{12} & b_{13} \\
b_{21} & b_{22} & b_{23} \\
b_{31} & b_{32} & b_{33}
\end{array}\right]\left[\begin{array}{ccc}
c & 0 & s \\
0 & 1 & 0 \\
-s & 0 & c
\end{array}\right]+\delta\left\{\begin{array}{l}
\mathrm{C}_{11}^{*}+\mathrm{C}_{12}^{*}+\mathrm{C}_{13}^{*} \\
\mathrm{C}_{21}^{*}+\mathrm{C}_{22}^{*}+\mathrm{C}_{23}^{*} \\
\mathrm{C}_{31}^{*}+\mathrm{C}_{32}^{*}+\mathrm{C}_{33}^{*}
\end{array}\right\}
$$

Here, $C_{11}^{*}, C_{12}^{*}$ etc. are elastic constants of the precipitate expressed in the Voigt notation. $c$ and $s$ are the short notations for $\cos \phi$ and $\sin \phi$, respectively. Under an uniaxial applied stress $\left(\sigma_{33}^{o}\right)$, the only non-zero component of $b_{i j}$ is $b_{33}$. For an isotropic elastic precipitate with $C_{i j k l}^{*}=f C_{i j k l}$, it is easy to establish that,

$$
\left\{\begin{array}{l}
\mathrm{C}_{11}^{*}+\mathrm{C}_{12}^{*}+\mathrm{C}_{13}^{*} \\
\mathrm{C}_{21}^{*}+\mathrm{C}_{22}^{*}+\mathrm{C}_{23}^{*} \\
\mathrm{C}_{31}^{*}+\mathrm{C}_{32}^{*}+\mathrm{C}_{33}^{*}
\end{array}\right\}=\frac{f E}{(1-2 \nu)}
$$

Therefore,

$$
\{B\}_{\phi}=\left\{\begin{array}{c}
\mathrm{b}_{33} \mathrm{~s}^{2}+\delta \frac{f E}{(1-2 \nu)} \\
\delta \frac{f E}{(1-2 \nu)} \\
\mathrm{b}_{33} \mathrm{c}^{2}+\delta \frac{f E}{(1-2 \nu)} \\
0 \\
-\mathrm{b}_{33} \mathrm{cs} \\
0
\end{array}\right\}
$$

The equivalent eigenstrain vector can then be simply obtained by inverting the matrix $[A]$,

$$
\{X\}_{\phi}=[A]^{-1}\{B\}_{\phi}=[\alpha]\{B\}_{\phi}
$$

For an isotropic elastic problem, the $6 \mathrm{x} 6$ matrix $[\alpha]$ is symmetric and can be expressed as,

$$
\{\alpha\}=\left[\begin{array}{cccccc}
\alpha_{11} & \alpha_{12} & \alpha_{13} & 0 & 0 & 0 \\
\alpha_{12} & \alpha_{22} & \alpha_{23} & 0 & 0 & 0 \\
\alpha_{13} & \alpha_{23} & \alpha_{33} & 0 & 0 & 0 \\
0 & 0 & 0 & \alpha_{44} & 0 & 0 \\
0 & 0 & 0 & 0 & \alpha_{55} & 0 \\
0 & 0 & 0 & 0 & 0 & \alpha_{55}
\end{array}\right]
$$

Then, 


$$
\{X\}_{\phi}=\left\{\begin{array}{c}
\alpha_{11} b_{33} s^{2}+\alpha_{13} b_{33} c^{2} \\
\alpha_{12} b_{33} s^{2}+\alpha_{23} b_{33} c^{2} \\
\alpha_{13} b_{33} s^{2}+\alpha_{33} b_{33} c^{2} \\
0 \\
-\alpha_{55} b_{33} c s \\
0
\end{array}\right\}+\delta \frac{f E}{(1-2 \nu)}\left\{\begin{array}{c}
\alpha_{11}+\alpha_{12}+\alpha_{13} \\
\alpha_{12}+\alpha_{22}+\alpha_{23} \\
\alpha_{13}+\alpha_{23}+\alpha_{33} \\
0 \\
0 \\
0
\end{array}\right\}
$$

The fictitious eigenstrains introduced in the equivalent inclusion method can be obtained as Mura (1987),

$$
\left\{\varepsilon^{*}\right\}_{\phi}=\{X\}_{\phi}-\left\{\varepsilon^{p}\right\}
$$

The elastic stresses inside the precipitate, resulting from the prescribed eigenstrains and the inhomogeneity, can be obtained as Mura (1987),

$$
\sigma_{i j}=C_{i j k l}\left(S_{k l m n} \varepsilon_{m n}^{* *}-\varepsilon_{k l}^{* *}\right)
$$

Thus,

$$
\begin{aligned}
& \sigma_{11}=\left[\alpha_{11} b_{33} s^{2}+\alpha_{13} b_{33} c^{2}+\delta \frac{f E\left(\alpha_{11}+\alpha_{12}+\alpha_{13}\right)}{1-2 \nu}\right]\left\{C_{11} S_{1111}+C_{12} S_{2211}+C_{13} S_{3311}-C_{11}\right\} \\
&+\left[\alpha_{12} b_{33} s^{2}+\alpha_{23} b_{33} c^{2}+\delta \frac{f E\left(\alpha_{12}+\alpha_{22}+\alpha_{23}\right)}{1-2 \nu}\right]\left\{C_{11} S_{1122}+C_{12} S_{2222}+C_{13} S_{3322}-C_{12}\right\} \\
&+\left[\alpha_{13} b_{33} s^{2}+\alpha_{33} b_{33} c^{2}+\delta \frac{f E\left(\alpha_{13}+\alpha_{23}+\alpha_{33}\right)}{1-2 \nu}\right]\left\{C_{11} S_{1133}+C_{12} S_{2233}+C_{13} S_{3333}-C_{13}\right\} \\
&-\left[\alpha_{55} b_{33} c s\right]\left\{2 C_{11} S_{1133}+2 C_{12} S_{2213}+2 C_{13} S_{3313}\right\}
\end{aligned}
$$

$$
\begin{aligned}
& \sigma_{22}=\left[\alpha_{11} b_{33} s^{2}+\alpha_{13} b_{33} c^{2}+\delta \frac{f E\left(\alpha_{11}+\alpha_{12}+\alpha_{13}\right)}{1-2 \nu}\right]\left\{C_{21} S_{1111}+C_{22} S_{2211}+C_{23} S_{3311}-C_{21}\right\} \\
&+\left[\alpha_{12} b_{33} s^{2}+\alpha_{23} b_{33} c^{2}+\delta \frac{f E\left(\alpha_{12}+\alpha_{22}+\alpha_{23}\right)}{1-2 \nu}\right]\left\{C_{21} S_{1122}+C_{22} S_{2222}+C_{23} S_{3322}-C_{22}\right\} \\
&+\left[\alpha_{13} b_{33} s^{2}+\alpha_{33} b_{33} c^{2}+\delta \frac{f E\left(\alpha_{13}+\alpha_{23}+\alpha_{33}\right)}{1-2 \nu}\right]\left\{C_{21} S_{1133}+C_{22} S_{2233}+C_{23} S_{3333}-C_{23}\right\} \\
&-\left[\alpha_{55} b_{33} c s\right]\left\{2 C_{21} S_{1133}+2 C_{22} S_{2213}+2 C_{23} S_{3313}\right\}
\end{aligned}
$$




$$
\begin{aligned}
& \sigma_{33}=\left[\alpha_{11} b_{33} s^{2}+\alpha_{13} b_{33} c^{2}+\delta \frac{f E\left(\alpha_{11}+\alpha_{12}+\alpha_{13}\right)}{1-2 \nu}\right]\left\{C_{31} S_{1111}+C_{32} S_{2211}+C_{33} S_{3311}-C_{31}\right\} \\
&+\left[\alpha_{12} b_{33} s^{2}+\alpha_{23} b_{33} c^{2}+\delta \frac{f E\left(\alpha_{12}+\alpha_{22}+\alpha_{23}\right)}{1-2 \nu}\right]\left\{C_{31} S_{1122}+C_{32} S_{2222}+C_{33} S_{3322}-C_{32}\right\} \\
&+\left[\alpha_{13} b_{33} s^{2}+\alpha_{33} b_{33} c^{2}+\delta \frac{f E\left(\alpha_{13}+\alpha_{23}+\alpha_{33}\right)}{1-2 \nu}\right]\left\{C_{31} S_{1133}+C_{32} S_{2233}+C_{33} S_{3333}-C_{33}\right\} \\
&-\left[\alpha_{55} b_{33} c s\right]\left\{2 C_{31} S_{1133}+2 C_{32} S_{2213}+2 C_{33} S_{3313}\right\}
\end{aligned}
$$

The applied stress vector in the local co-ordinate system of the precipitate is,

$$
\left\{S^{o}\right\}_{\phi}=\left\{\begin{array}{c}
\sigma_{33}^{o} \mathrm{~s}^{2} \\
0 \\
\sigma_{33}^{o} \mathrm{c}^{2} \\
0 \\
-\sigma_{33}^{o} \mathrm{cs} \\
0
\end{array}\right\}
$$

Using the definition of $b_{i j}$, it can be shown that,

$$
\begin{aligned}
b_{33}= & (1-f)\left[C_{3311} \varepsilon_{11}^{o}+C_{3322} \varepsilon_{22}^{o}+C_{3333} \varepsilon_{33}^{o}\right] \\
& =(1-f)\left[C_{1111}-2 \nu C_{1133}\right] \frac{\sigma_{33}^{o}}{E}=(1-f) \sigma_{33}^{o}
\end{aligned}
$$

The total potential energy of the elastic system, measured with respect to the loaded configuration, can be expressed as Mura (1987)

$$
G^{*}=-\frac{1}{2}\left\{S^{o}\right\}^{T}\left\{\varepsilon^{*}\right\}_{\phi}-\frac{1}{2}\left(\sigma_{11}+\sigma_{22}+\sigma_{33}\right) \delta-\sigma_{33}^{o} \delta
$$

The orientation of the precipitate (for a given shape) that minimizes the total potential energy can be obtained as,

$$
\frac{\partial G^{*}}{\partial \phi}=0
$$

After some lengthy algebraic rearrangements, the following equation for $\phi$ can be obtained,

$$
\tan ^{2} \phi=\frac{\left(\alpha_{33}-\alpha_{13}-\alpha_{55}\right)}{\left(\alpha_{11}-\alpha_{13}-\alpha_{55}\right)}+\frac{\delta \eta}{\sigma_{33}^{o}\left(\alpha_{11}-\alpha_{13}-\alpha_{55}\right)} \sec ^{2} \phi
$$

Here,

$$
\begin{array}{r}
\eta=\frac{1}{2}\left[\frac{f E}{(1-f)(1-2 \nu)}\left\{\alpha_{23}+\alpha_{33}-\alpha_{11}-\alpha_{12}\right\}\right]-\frac{\left(\alpha_{11}-\alpha_{13}\right) \bar{A}}{2} \\
-\frac{\left(\alpha_{12}-\alpha_{23}\right) \bar{B}}{2}-\frac{\left(\alpha_{13}-\alpha_{33}\right) \bar{C}}{2}
\end{array}
$$


and,

$$
\begin{array}{r}
\bar{A}=S_{1111}\left(C_{11}+C_{21}+C_{31}\right)+S_{2211}\left(C_{12}+C_{22}+C_{32}\right)+ \\
S_{3311}\left(C_{13}+C_{23}+C_{33}\right)-\left(C_{11}+C_{21}+C_{31}\right) \\
\bar{B}=S_{1122}\left(C_{11}+C_{21}+C_{31}\right)+S_{2222}\left(C_{12}+C_{22}+C_{32}\right)+ \\
S_{3322}\left(C_{13}+C_{23}+C_{33}\right)-\left(C_{12}+C_{22}+C_{32}\right) \\
\bar{C}=S_{1133}\left(C_{11}+C_{21}+C_{31}\right)+S_{2233}\left(C_{12}+C_{22}+C_{32}\right)+ \\
S_{3333}\left(C_{13}+C_{23}+C_{33}\right)-\left(C_{13}+C_{23}+C_{33}\right)
\end{array}
$$

Perhaps a more practical form of eq.(A-17) is,

$$
\left(\alpha_{11}-\alpha_{13}-\alpha_{55}\right) \sigma_{33}^{o} \sin ^{2} \phi-\left(\alpha_{33}-\alpha_{13}-\alpha_{55}\right) \sigma_{33}^{o} \cos ^{2} \phi-\delta \eta=0
$$

For an elastic inhomogeneity, $(\delta=0)$, the orientation corresponding to minimum potential energy is given by the following expression,

$$
\tan \phi=\sqrt{\frac{\left(\alpha_{33}-\alpha_{13}-\alpha_{55}\right)}{\left(\alpha_{11}-\alpha_{13}-\alpha_{55}\right)}}
$$

The usefulness of the proposed analytical scheme becomes apparent when we consider some special cases. For simple geometries, such as disc and needle shaped precipitates, closed-form expressions describing the relation between applied uniaxial stress and the orientation of precipitate can be obtained.

\section{Appendix A.2 Disc shaped precipitate under uniaxial stress}

For a disc shaped precipitate in an isotropic matrix, the non-zero components of Eshelby's tensor are Mura (1987),

$$
S_{1313}=S_{3131}=S_{2323}=S_{3232}=\frac{1}{2} ; \quad S_{3311}=S_{3322}=\frac{\nu}{1-\nu} ; \quad S_{3333}=1
$$

On substituting these values of $S_{i j k l}$ in the left hand side of eq.(A-1) and using the Voigt notation, the components of the $[A]$ matrix can be obtained. After inverting the $[A]$ matrix and carrying out some algebraic simplifications, the components $\alpha_{i j}$ are,

$$
\begin{array}{r}
\alpha_{11}=\alpha_{22}=\frac{1}{E} ; \quad \alpha_{33}=\frac{(1+\nu)(1-2 \nu)+2 \nu^{2} f}{E f(1-\nu)} \\
\alpha_{12}=\alpha_{13}=\alpha_{23}=-\frac{\nu}{E} ; \quad \alpha_{55}=\frac{(1+\nu)}{E f}
\end{array}
$$

Therefore, 


$$
\begin{array}{r}
\left(\alpha_{11}-\alpha_{13}-\alpha_{55}\right)=\frac{(1+\nu)(f-1)}{E f} ; \quad\left(\alpha_{33}-\alpha_{13}-\alpha_{55}\right)=\frac{\nu(1+\nu)(f-1)}{E f(1-\nu)} \\
\left(\alpha_{23}+\alpha_{33}-\alpha_{11}-\alpha_{12}\right)=\frac{(1-f)\left(1-\nu-2 \nu^{2}\right)}{E f(1-\nu)}
\end{array}
$$

Also,

$$
\bar{A}=\bar{B}=\left(C_{11}+2 C_{12}\right)\left(S_{3311}-1\right)=-\frac{E}{(1-\nu)} ; \quad \bar{C}=0
$$

and

$$
\eta=\frac{(1+\nu)}{(1-\nu)}
$$

On substitution of eqs.(A-27)-(A-29) in eq.(A-23), the following expression can be obtained,

$$
\sigma_{33}^{o}\left\{\sin ^{2} \phi-\frac{\nu}{1-\nu} \cos ^{2} \phi\right\}-\frac{\delta f E}{(f-1)(1-\nu)}=0
$$

For the case of an elastic inhomogeneity $(\delta=0)$, the orientation corresponding to minimum potential energy is given by the following equation,

$$
\tan \phi=\sqrt{\frac{\nu}{1-\nu}}
$$

\section{Appendix A.3 Needle shaped precipitate under uniaxial stress}

For a needle shaped precipitate, the non-zero components of Eshelby's tensor are Mura (1987),

$$
\begin{gathered}
S_{1111}=S_{2222}=\frac{5-4 \nu}{8(1-\nu)} ; \quad S_{1122}=S_{2211}=\frac{4 \nu-1}{8(1-\nu)} ; \quad S_{2233}=S_{1133}=\frac{\nu}{2(1-\nu)} \\
S_{1212}=\frac{3-4 \nu}{8(1-\nu)} ; \quad S_{2323}=S_{3232}=S_{1313}=S_{3131}=\frac{1}{4}
\end{gathered}
$$

Following the procedure described for a disc shaped precipitate, the resulting expressions for a needle shaped precipitate are,

$$
\begin{aligned}
\left(\alpha_{33}-\alpha_{13}-\alpha_{55}\right) & =\frac{(1+\nu)(f-1)}{E(f+1)} ; \\
\left(\alpha_{11}-\alpha_{13}-\alpha_{55}\right) & =\frac{(1-f)(\nu+1)\left(4 f^{2} \nu^{2}-3 f^{2} \nu-3 f \nu+f+4 \nu^{2}-4 \nu+1\right)}{E(f+1)(1+f-2 \nu)(3 f-4 f \nu+1)}
\end{aligned}
$$

Also,

$$
\bar{A}=\bar{B}=\frac{E}{2(\nu-1)} ; \quad \bar{C}=\frac{E}{(\nu-1)}
$$


and

$$
\eta=-\frac{(\nu+1) f}{(1+f-2 \nu)}
$$

Substitution of eqs.(A-33)-(A-35) in eq.(A-23), the following expression can be obtained,

$$
\sigma_{33}^{o}\left\{\bar{D} \sin ^{2} \phi+(1+f-2 \nu) \cos ^{2} \phi\right\}-\frac{\delta f E(1+f)}{(f-1)}=0
$$

Here,

$$
\bar{D}=\frac{4 f^{2} \nu^{2}-3 f^{2} \nu-3 f \nu+f+4 \nu^{2}-4 \nu+1}{3 f-4 f \nu+1}
$$

For an elastic inhomogeneity $(\delta=0)$, the orientation corresponding to minimum potential energy is given by the following equation,

$$
\tan \phi=\sqrt{\frac{-(1+f-2 \nu)}{\bar{D}}}
$$

For $f \leq 0.645$, no real analytical solution exists for the orientation of a needle shaped precipitate that minimizes the potential energy.

\section{Appendix B Potential energy of an elastic inhomogene- ity under biaxial stress}

We consider here, an elastic inhomogeneity in an isotropic elastic matrix. Applied stress is assumed to be biaxial, acting in the $x_{1}-x_{3}$ plane. Following the notation used in Appendix-A, the vector $\{B\}$ in the local co-ordinate system of the precipitate can be expressed as,

$$
\{B\}_{\phi}=\left\{\begin{array}{c}
\mathrm{b}_{11} \mathrm{c}^{2}+\mathrm{b}_{33} \mathrm{~s}^{2} \\
0 \\
\mathrm{~b}_{11} \mathrm{~s}^{2}+\mathrm{b}_{33} \mathrm{c}^{2} \\
0 \\
\left(\mathrm{~b}_{11}-\mathrm{b}_{33}\right) \mathrm{cs} \\
0
\end{array}\right\}
$$

The equivalent eigenstrain vector can be obtained as,

$$
\{X\}_{\phi}=\left\{\begin{array}{c}
\alpha_{11}\left(b_{11} c^{2}+b_{33} s^{2}\right)+\alpha_{13}\left(b_{11} s^{2}+b_{33} c^{2}\right) \\
\alpha_{12}\left(b_{11} c^{2}+b_{33} s^{2}\right)+\alpha_{23}\left(b_{11} s^{2}+b_{33} c^{2}\right) \\
\alpha_{13}\left(b_{11} c^{2}+b_{33} s^{2}\right)+\alpha_{33}\left(b_{11} s^{2}+b_{33} c^{2}\right) \\
0 \\
\alpha_{55}\left(b_{11}-b_{33}\right) c s \\
0
\end{array}\right\}
$$


The external stress vector in the local co-ordinate system of the precipitate is,

$$
\left\{S^{o}\right\}_{\phi}=\left\{\begin{array}{c}
\left(\sigma_{11}^{o} \mathrm{c}^{2}+\sigma_{33}^{o} \mathrm{~s}^{2}\right) \\
0 \\
\left(\sigma_{11}^{o} \mathrm{~s}^{2}+\sigma_{33}^{o} \mathrm{c}^{2}\right) \\
0 \\
\left(\sigma_{11}^{o}-\sigma_{33}^{o}\right) \mathrm{cs} \\
0
\end{array}\right\}
$$

Denoting,

$$
\beta=\frac{\sigma_{11}^{o}}{\sigma_{33}^{o}}
$$

and using the definition of $b_{i j}$, it can be shown that,

$$
\begin{gathered}
b_{11}=(1-f)\left[C_{1111} \varepsilon_{11}^{o}+C_{1122} \varepsilon_{22}^{o}+C_{1133} \varepsilon_{33}^{o}\right]=(1-f) \beta \sigma_{33}^{o} \\
b_{33}=(1-f)\left[C_{3311} \varepsilon_{11}^{o}+C_{3322} \varepsilon_{22}^{o}+C_{3333} \varepsilon_{33}^{o}\right]=(1-f) \sigma_{33}^{o}
\end{gathered}
$$

The total potential energy of an elastic inhomogeneity can be expressed as Mura (1987)

$$
G^{*}=-\frac{1}{2}\left\{S^{o}\right\}^{T}\left\{\varepsilon^{*}\right\}_{\phi}
$$

The orientation of the precipitate that minimizes the total potential energy is,

$$
\frac{\partial G^{*}}{\partial \phi}=0
$$

After some algebraic simplifications, the following equation for $\phi$ can be obtained,

$$
\tan ^{2} \phi=\frac{\left(\alpha_{33}-\alpha_{13}-\alpha_{55}\right)+\beta\left(2 \alpha_{13}-\alpha_{11}-\alpha_{33}+2 \alpha_{55}\right)+\beta^{2}\left(\alpha_{11}-\alpha_{13}-\alpha_{55}\right)}{\left(\alpha_{11}-\alpha_{13}-\alpha_{55}\right)+\beta\left(2 \alpha_{13}-\alpha_{11}-\alpha_{33}+2 \alpha_{55}\right)+\beta^{2}\left(\alpha_{33}-\alpha_{13}-\alpha_{55}\right)}
$$

For the special case of equi-biaxial stress $(\beta=1)$, the external stress vector, $\left\{S^{o}\right\}_{\phi}$, the equivalent eigenstrain vector, $\{X\}_{\phi}$, and, hence, the total potential energy of an elastic inhomogeneity is independent of its orientation. Using the expressions for $\alpha_{i j}$, provided in eq.(A-26), it is fairly straightforward to establish the following relation for a disc shaped precipitate,

$$
\left(2 \alpha_{13}-\alpha_{11}-\alpha_{33}+2 \alpha_{55}\right)=-\frac{(1+\nu)(f-1)}{E f(1-\nu)}
$$

On substitution of eq.(A-27) and eq.(B-10) in eq.(B-9) and after some algebraic simplifications, the expression describing the effect of stress biaxiality on the orientation of a disc shaped elastic inhomogeneity can be obtained as,

$$
\tan \phi=\sqrt{\frac{\nu-(1-\nu) \beta}{1-\nu-\nu \beta}}
$$


Similar to the case of a uniaxial stress, the orientation minimizing the potential energy of a disc shaped inhomogeneity under bi-axial stress is independent of the elastic mismatch between the matrix and precipitate. For a typical value of $\nu=0.3$, a real solution for $\phi$ exists provided $\beta<0.43$.

\section{References}

Bai, J.B., Ji, N., Gilbon, D., Prioul, C., Francois, D. (1994). Hydride embrittlement in Zircaloy4 plate: Part II. Interaction between the tensile stress and the hydride morphology, Metall. Mater. Trans. A, 25, 1199-1208.

Bair, J., Zaeem, M.A., Tonks, M. (2015). A review on hydride precipitation in zirconium alloys, J. Nucl. Mater., 466, 12-20.

Bair, J., Zaeem, M.A. (2017). Effects of applied strain on formation, shape evolution, and reorientation of multiphase zirconium hydrides: A multiphase field modeling study, arXiv preprint arXiv:1706.01097.

Carpenter, G.J.C. (1973). The dilatational misfit of zirconium hydrides precipitated in zirconium, J. Nucl. Mater., 48, 264-266.

Christian, J.W. (2002). The theory of transformations in metals and alloys - Part I, $3^{\text {rd }}$ edition, Pergamon.

Chu, H.C., Wu, S.K., Kuo, R.C. (2008). Hydride reorientation in Zircaloy-4 cladding, J. Nucl. Mater., 373, 319-327.

Cinbiz, M.N., Koss, D.A., Motta, A.T. (2016). The influence of stress state on the reorientation of hydrides in a zirconium alloy, J. Nucl. Mater., 477, 157-164.

Ells, C.E. (1970). The stress orientation of hydride in Zirconium alloys, J. Nucl. Mater., 35, 306-315.

Eshelby, J.D. (1957). The determination of the elastic field of an ellipsoidal inclusion, and related problems, Proc. R. Soc.(A), 241, 376-396.

Fisher, E.S., Renken, C.J. (1964). Single-crystal elastic moduli and the hcp $\rightarrow$ bcc transformation in Ti, Zr, and Hf, Phys. Rev. A, 135, 482-494.

Gill, S.P.A., Cocks, A.C.F. (2006). An analytical model for the energetics of quantum dots: beyond the small slope assumption, Proc. R. Soc. A., 642, 3523-3553.

Han, G.M., Zhao, Y.F., Zhou, C.B., Lin, De-Ye, Zhu, X.Y., Zhang, J., Hu, S.Y., Song, H.F. (2019). Phase-field modeling of stacking structure formation and transition of $\delta$-hydride precipitates in zirconium, Acta Mater., 165, 528-546.

Kato, M., Fujii, T., Onaka, S. (1996). Elastic states of inhomogeneous spheroidal inclusions, Mater. Trans., 37, 314-318. 
Leger, M., Donner, A. (1985). The Effect of stress on orientation of hydrides in Zirconium alloy pressure tube materials, Can. Metall. Q., 24, 235-243.

Leitch, B.W., Puls, M.P. (1992). Finite element calculations of the accommodation energy of a misfitting precipitate in an elastic-plastic matrix, Metall. Trans. (A), 23, 797-806.

Massih, A.R., Jernkvist, L.O. (2009). Stress orientation of second-phase in alloys: Hydrides in zirconium alloys, Comput. Mater. Sci., 46, 1091-1097.

Mori, T., Cheng, P.C., Kato, M., Mura, T. (1978). Elastic strain energies of precipitates and periodically distributed inclusions in anisotropic media, Acta metall., 26, 1435-1441.

Motta, A.T., Capolungo, L., Chen, L.Q., Cinbiz, M.N., Daymond, M.R., Koss, D.A. Lacroix, E., Pastore, G., Simon, P.C.A., Tonks, M.R., Wirth, B.D., Zikry, M.A. (2019). Hydrogen in zirconium alloys: a review, J. Nucl. Mater., 518, 440-460.

Mura, T. (1987). Micromechanics of defects in solids, $2^{\text {nd }}$ edition, Martinus Nijhoff Publishers.

Northwood, D.O., Kosasih, U. (1983). Hydrides and delayed hydrogen cracking in zirconium and its alloys, Int. Met. Rev., 28: 92-121.

Onaka, S., Kato, M., Fujii, T. (1995). The elastic strain energy of a coherent inclusion with deviatoric misfit strains, Mech. Mater., 20, 329-336.

Perovic, V., Purdy, G.R., Brown, L.M. (1981). Autocatalytic nucleation and elastic stabilization of linear arrays of plate-shaped precipitates, Acta Metall., 29, 889-902.

Pineau, A. (1976). Influence of uniaxial stress on the morphology of coherent precipitates during coarsening-Elastic energy considerations, Acta Metall., 24, 549-554.

Puls, M.P. (2009). Review of the thermodynamic basis for models of delayed hydride cracking rate in zirconium alloys, J. Nucl. Mater., 393, 350-367.

Puls, M.P. (2012). The effect of hydrogen and hydrides on the integrity of zirconium alloy components Delayed Hydride Cracking, Springer.

Sato, A., Mori, T., Tanaka, Y. 1979. Stress distribution around an inclusion in an Fe-N single crystal, Acta Metall., 27, 131-136.

Sharma, R.K., Tewari, A., Singh, R.N., Kashyap, B.P. (2018). Optimum shape and orientation of $\delta$-hydride precipitate in $\alpha$-zirconium matrix for different temperatures. J. Alloys Compd., $742,804-813$.

Singh, R.N., Stahle, P., Sills, L.B., Ristmanaa, M., Banerjee, S. (2008). $\delta$-hydride habit plane determination in $\alpha$-zirconium at $298 \mathrm{~K}$ by strain energy minimization technique, Defect Diffus. forum, 279, 105-110.

Taylor, J.E., Cahn, J.W. (1986). A cusp singularity in surfaces that minimizes an anisotropic surface energy, Science, 233, 548-551.

Une, K., Nogita, K., Ishimoto, S., Ogata, K. (2004). Crystallography of Zirconium hydrides in recrystallized Zircaloy-2 fuel cladding by electron backscatter diffraction, J. Nucl. Sci. Technol., 41, 731-740. 